\title{
Dynamics of coherent structures and turbulence of plasma drift waves
}

\author{
C. Ferro Fontán \\ Instituto de Física del Plasma,* \\ Departamento de Física, Facultad de Ciencias Exactas y Naturales, Ciudad Universitaria, Pabellón I, \\ 1428 Buenos Aires, Argentina \\ A. Verga ${ }^{\dagger}$ \\ Equipe Turbulence Plasma, ${ }^{\ddagger}$ Institut Méditerranéen de Technologie, \\ F-13451, Marseille Cedex 20, France
}

(Received 11 April 1995; revised manuscript received 26 July 1995)

\begin{abstract}
A simple model based on the Hasegawa-Mima equation is used to study dipole vortex interactions and turbulence of electrostatic drift waves in a plasma. It is shown that if nonlinear effects are important during vortex collisions, dipoles are broken into monopoles. Nonadiabatic effects also affect dipole behavior, which can be destroyed by the instability of emitted waves (dipole vortex radiation). Simulations of turbulence in both decaying and driven cases show the appearance of long-lived monopole structures. These coherent structures contribute to stop the cascade of energy to large scales, and then to reach a self-organized stationary state. Some numerical evidence is done that Hasegawa-Mima turbulence has a long-time behavior that is much richer than the thermodynamic equilibrium state observed in two-dimensional hydrodynamics. In driven turbulence, an important departure from Gaussian statistics of vorticity fluctuations is found, giving some indication of intermittency. Using various analyzing techniques, in particular the proper orthogonal decomposition, we show that the turbulence can be characterized by a field of coherent structures, which dominates the dynamics of the system, and random waves interacting weakly with the coherent structures.
\end{abstract}

PACS number(s): 52.35.Kt, 52.35.Ra, 52.65.-y

\section{INTRODUCTION}

One of the most interesting and intriguing features of turbulence is its tendency to some kind of selforganization [1]. The appearance of coherent structures in two-dimensional (2D) [2-5] and three- [6,7] dimensional fluid and magnetohydrodynamic turbulence $[8,9]$ is a well-established phenomenon from both experimental and numerical studies (see, e.g., Ref. [10] for an account on the concept of coherent structure from an experimental point of view). This approach to an organized state, by the formation of coherent structures, is conceptually different from the traditional inverse cascade hypotheses $[11,12]$.

It is the purpose of the present paper to study the properties of such coherent structures in the case of electrostatic turbulence in plasmas. A comprehensive review on drift plasma turbulence can be found in Ref. [13]. In particular, we are interested in understanding the mechanisms of saturation, if they exist, of the inverse cascade. One possibility, without introducing a supplemen-

\footnotetext{
${ }^{*}$ Consejo Nacional de Investigaciones Científicas y TécnicasUniversidad de Buenos Aires, Buenos Aires, Argentina.

${ }^{\dagger}$ Electronic address: verga@utp.imt-mrs.fr

${ }^{\ddagger}$ URA 773, Centre National de la Recherche ScientifiqueUniversité de Provence, Marseille, France.
}

tary dissipation at large scales, is just the generation of vortex structures. Indeed, the generic nonlinearity in plasma (or fluid) two-dimensional flows is a Poisson bracket between some generalized vorticity $\Omega$ and the stream function $\phi$ (or the electric potential in plasma). The coherent structure is a solution of an equation of the form

$$
\Omega=F(\phi),
$$

which precisely weakens the nonlinear term, suppressing by the way the cascade source. The simplest model of electrostatic waves in a plasma is given by an evolution equation for the potential $\phi(x, y, t)$ taking the form

$$
(1+L) \frac{\partial}{\partial t} \phi+\frac{\partial}{\partial y} \phi+[\phi, L \phi]=-\nu \Delta^{2} \phi,
$$

where the operator $L$ is just $-\Delta$ for the Hasegawa-Mima $[14,15]$ model, and

$$
L=-\Delta+\delta_{0}\left(c_{0}+\Delta\right) \frac{\partial}{\partial y}
$$

for the Terry and Horton model [16-18], where nonadiabatic electron response is taken into account through the terms in $\delta_{0}$. The terms in $\nu$ add some large-wave-vector dissipation, and on the other hand if $c_{0} \neq 0$ dissipation is also present at small wave vectors. Units were chosen in such a way as to obtain the drift velocity $v_{d}=1$. Length is measured in Larmor radius $\rho_{s}$, time in $\left(L_{n} / \omega_{c}\right)$, where (c) 1995 The American Physical Society 
$L_{n}$ is the density gradient length and $\omega_{c}$ is the cyclotron frequency, the potential fluctuations $\phi$ are measured in units of $\left(T_{e} / e\right)\left(\rho_{s} / L_{n}\right)$.

Equation (1.2) was introduced in the study of drift waves in a tokamak edge plasma. It can be interpreted as a simplification of the Hasegawa-Wakatani system of coupled equations for the density and potential fluctuations [19], where only the unstable branch of the dispersion relation was retained. This unstable branch describes the phase shift between density and potential fluctuations. Certainly this equation is too simple to describe the complicated processes present in the tokamak, but it contains the essential ingredients that play a fundamental role in plasma turbulence, and is then an appropriate model to investigate the physics of these processes.

Equation (1.2) contains many interesting features: linear dispersive waves and a range of unstable modes giving a source of free energy; small scale dissipation providing a sink of energy to the system; a "vector" nonlinearity, the Poisson bracket [, ], responsible for the generation of coherent structures. Instability, damping, and nonlinearity, acting at different spatial and temporal scales can produce a very rich variety of phenomena, characteristic of plasma turbulence.

We can see that defining a generalized vorticity $\Omega=$ $\Delta \phi-\phi+x$ the Hasegawa-Mima equation can be written in the form

$$
\frac{\partial}{\partial t} \Omega+[\phi, \Omega]=0
$$

and then Eq. (1.1) gives the stationary solutions. Equation (1.4) is much like the Euler equation of hydrodynamics, in which case $\Omega=\Delta \phi$ is the usual vorticity with $-\phi$ the current function.

The understanding of coherent structures is essential for the interpretation of turbulence from a dynamical point of view. In addition, coherent structures modify the statistical properties of the flows and in some regimes dominate their dynamics $[13,20,21]$. Stationary vortex solutions of the Hasegawa-Mima equation are well known [21-24]. Although dipole vortices were extensively studied, their relevance as elementary constituents of turbulence, is dubious $[18,24,25]$. However, the investigation of dipole collisions and stability may give some insight in the behavior of nonlinear structures (see Sec. II). It is important to point out that dipoles are the only (simple and localized) fully nonlinear solution of (1.2). The study of the dynamics of dipoles provides, in a controlled manner, a way of analyzing how perturbations may affect more general vortices. In Sec. II we investigated dipole interactions in both conservative and nonadiabatic regimes.

We also studied in this paper the turbulent regime for pure decaying and driven-damped equations. A comparison of some related hydrodynamics systems is made. One important question addressed in Sec. III is whether Hasegawa-Mima decaying turbulence evolves in a similar way as 2D hydrodynamics [26,27], in the sense that the system approaches a state of thermodynamic equilibrium. Both Euler and Hasegawa-Mima equations can be put in a form of frozen generalized vorticity equation; both equations have an infinity of integrals of motion. However, there are two important differences: (i) the Hasegawa-Mima equation has a natural scale length (the Larmor radius, which gives rise to the term in $\phi$ in the generalized vorticity $\Omega=\Delta \phi-\phi+x$ ) and (ii) it allows for linear dispersive waves (related to the nonlocalized term of the generalized vorticity $\Omega$. As we will see these facts can affect the long-time evolution of the system.

In two-dimensional driven-damped electrostatic driftwave turbulence the first problem is to determine if a stationary state is reached. Indeed, for the simple model (1.2), where instability at intermediate and low wave numbers and "inverse" cascade work together, the question arises of the saturation of energy as the system evolves [28]. We performed (Sec. IV) high-resolution, long-time simulations of the Terry and Horton model, to investigate this stationary state, which is effectively realized. In particular, we analyzed its statistical properties using different techniques, as the proper orthogonal decomposition (POD) [29-31] and conditional averaging [32]. Moreover, in relation to recent experimental evidence on intermittency in edge plasma [33], we studied the probability distribution of potential and vorticity fluctuations.

Before detailing our simulations in the following sections, we give some technical elements about the code used. The numerical integration of (1.2) uses an antialiased pseudospectral method [34] on a periodic square mesh of typical size $256^{2}$. The time stepping is performed using an improved version of the Gazdag leap-frog corrected method [35]. Our method integrates exactly the linear part of the equations, which is essential to correctly describe unstable systems. Errors are monitored using the energy balance equation, the time step was chosen in order to keep energy balance correct to a factor of $10^{-6}$; the typical time step is $10^{-3}-10^{-4}$. We found this requirement necessary to obtain reliable and reproducible results.

\section{VORTEX INTERACTIONS}

The Hasegawa-Mima equation admits a class of localized stationary solutions in the form of multipoles $[13,24,36]$. The simplest dipole solution we used in the simulations is given by

$$
\phi(r, \theta)= \begin{cases}{\left[B+C J_{1}(p r)\right] \cos \theta} & \text { for } r<r_{0} \\ A K_{1}(k r) & \text { for } r>r_{0}\end{cases}
$$

where $J, K$ are Bessel functions and $r^{2}=x^{2}+(y-v t)^{2}$, $\theta$ are polar coordinates in the reference frame of dipole velocity $v$

$$
A=v r_{0} / K_{1}\left(k r_{0}\right), \quad B=v r_{0}\left(1+k^{2} / p^{2}\right),
$$

and

$$
C=v r_{0} k^{2} / p^{2} J_{1}\left(p r_{0}\right) .
$$

Moreover, $k=(1-1 / v)^{1 / 2}$ and $p$ are related by a "nonlinear" dispersion relation 


$$
\frac{K_{2}\left(k r_{0}\right)}{k K_{1}\left(k r_{0}\right)}=\frac{-J_{2}\left(p r_{0}\right)}{p J_{1}\left(p r_{0}\right)}
$$

Dipoles are then specified by the two parameters velocity $v$ and size $r_{0}$. It is worth noticing that a function in the form of a plane wave

$$
\phi(x, y, t)=A \exp \left(-i \omega_{k} t+i \mathbf{k} \cdot \mathbf{r}\right),
$$

where $\omega_{k}$ satisfies the dispersion relation, is a solution of the Hasegawa-Mima equation, and more generally a solution of (1.2). This property that an isolated linear mode is a full "nonlinear" solution is specific of the Poisson bracket form, and in contrast to the usual steeping effect of the scalar (as in Korteweg-de Vries equation) nonlinearity. As a consequence, an initial isolated mode, even if it is unstable, does not couple with other modes, and its evolution is trivial.

In this section we will study the interaction of dipoles to get some insight into the dynamics and properties of coherent structures. We separate the two cases of the pure conservative Hasegawa-Mima equation and its generalization by Terry and Horton.

\section{A. Dipole collisions in the conservative case}

One interesting numerical result about dipole collisions is that they can behave as solitons, as shown by Makino, Kamimura, and Taniuti [22]. They used dipoles of respective velocities $v_{+}=3$ and $v_{-}=-5$; and of the same size $r_{0}=0.5$, the dipole amplitude is then around 3 (using units where the drift velocity is equal to 1 ). After the head-on collision, the dipoles recovered their initial shape and moved again as translating steady solutions. However, in an overtaking collision of dipoles with unequal radii, McWilliams and Zabusky [23] observed their merging. These contrasted results show that dipoles are not exactly solitons in integrable systems, and that the question of their stability is open $[25,37]$. In fact, in most turbulence simulations, spontaneous generation of dipoles is not observed, and rather monopolar structures are present. We study the interaction of dipoles in the Hasegawa-Mima case for a wide range of parameters. One characteristic result is that dipole collisions can be classified using a parameter

$$
\Gamma=\frac{\tau_{\mathrm{NL}}}{\tau_{\mathrm{c}}}
$$

which is the ratio of two characteristic times, a time related to the nonlinearity, the vortex turnover $\tau_{\mathrm{NL}}=$ $r_{0}^{2} /|\phi|$, and a collision time $\tau_{c}=\Delta r_{0} / \Delta v$ where $\Delta r_{0}$ is the cross "section" and $\Delta v$ the relative velocity, $|\phi|$ is the amplitude of the vortex. The parameter $\Gamma$ is a measure of the role of the nonlinearity during the collision time. When $\Gamma \gg 1$, as in the Makino, Kamimura, and Tamiuti, case, the collision is almost elastic (solitonlike). In the opposite case, $\Gamma \ll 1$, nonlinearity is strong during the collision. A simulation with $\Gamma \ll 1$ is presented in Fig. 1. Initially two dipoles are separated wide apart, the box size is $55 \times 55$ and the distance between the dipoles is 20. Their parameters are $v_{+}=2, r_{+}=6$, and $v_{-}=-1, r_{-}=6$, respectively. Then their relative velocity is rather low [the region $0<v<1$ is forbidden
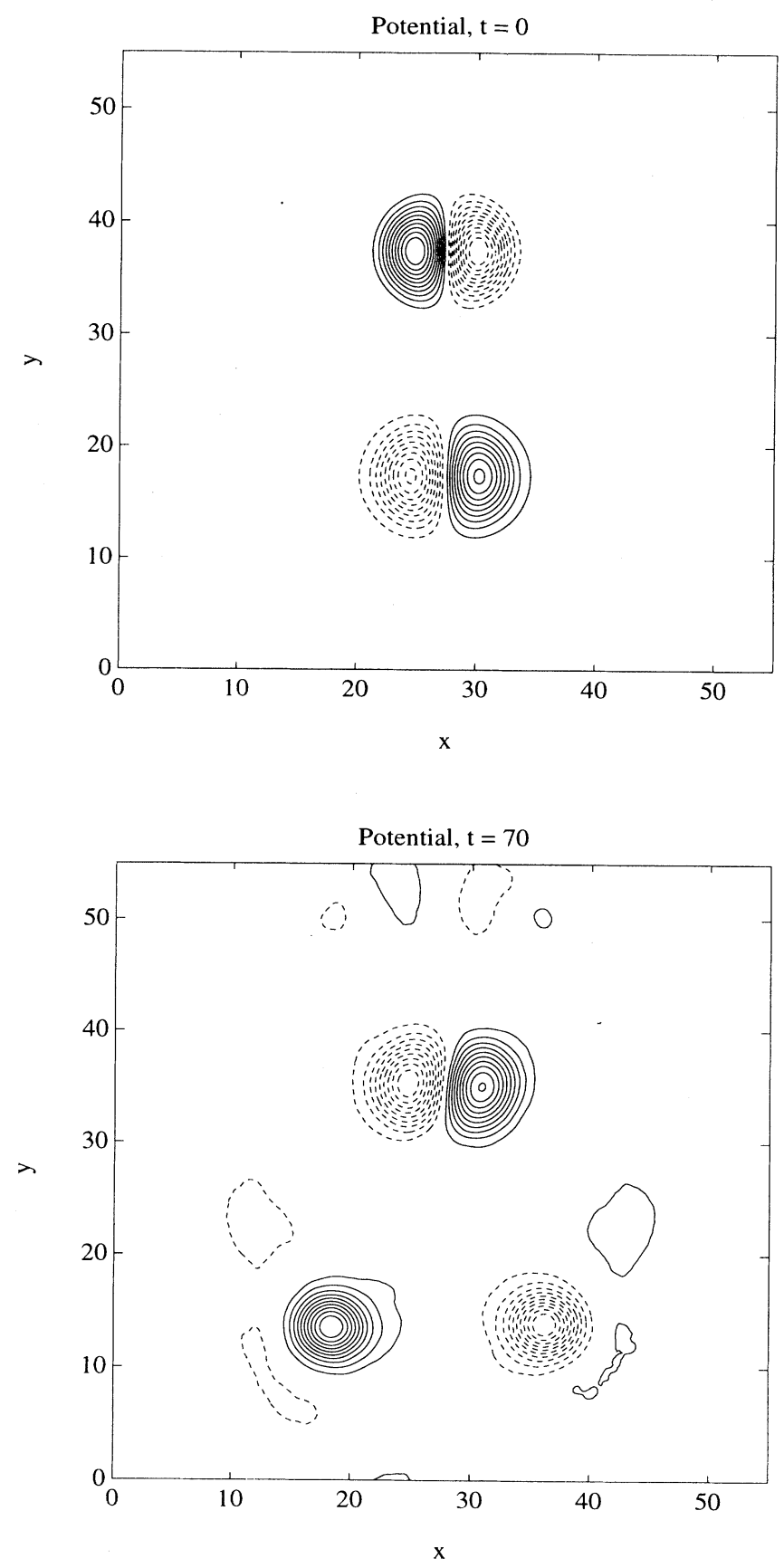

FIG. 1. Interaction of two slow and high amplitude dipoles in the Hasegawa-Mima case. Contour plots show the system before (top), $t=0$, and after the collision (bottom), $t=70$, where two monopoles were formed. This contrasts with the solitonlike behavior of the interaction of fast dipoles. The dipole parameters are $v_{+}=2, r_{+}=6, v_{-}=-1, r_{-}=6$. 
to dipoles, $k=(1-1 / v)^{1 / 2}$ becomes imaginary in this region], and their amplitudes are about 55, thus a rough estimate of $\Gamma$ is $\Gamma \approx 0.1$. Both initial dipoles are modified by their interaction. The positive velocity dipole $\left(D_{+}\right)$ separates into its two components during the collision, but they join together again, after the collision. This new dipole $\left(D_{+}^{\prime}\right)$ is slightly different from the initial one; its amplitude and size are decreased. The other dipole, traveling with a negative velocity $\left(D_{-}\right)$splits, after the collision, into two monopoles $\left(M_{+}\right)$(the sign index denotes the translation direction $\pm \hat{\mathbf{y}})$ :

$$
D_{+}+D_{-} \rightarrow M_{+1}+M_{+2}+D_{+}^{\prime}+\text { (radiation). }
$$

It is easy to identify the newly formed structures as monopoles; their velocity was inverted with respect to that of the parent dipole, and moreover, both positive and negative vortices travel at a forbidden speed for dipoles, in the region where linear waves can propagate. They also have a slight tendency to separate. While monopoles translate, they leave a wake of drift waves. The fact that during the collision some amount of radiation is emitted might be fundamental to the "irreversible" or inelastic character of the collision. Although isolated dipoles and linear waves interact weakly, the translation velocity of dipoles is larger than the wave phase velocity, during collision time dipoles are strongly perturbed, and in particular their velocities can enter the region of drift waves, allowing then the interaction.

In summary, for $\Gamma \gg 1$ dipoles are rather robust and collisions are elastic, and for $\Gamma \ll 1$ breaking of dipoles and formation of monopoles appears to be the common case. We point out that we investigated these processes for a variety of dipole parameters, obtaining the picture just described, provided $\Gamma$ is different from one. Precisely for $\Gamma \approx 1$ one may expect an interaction process much more complex. We choose dipole parameters to obtain a $\Gamma$ slightly smaller than $1(\Gamma \approx 0.7): v_{+}=3$, $r_{+}=1.44, v_{-}=-5, r_{-}=1.13 ;$ the amplitudes are about 9 . Figure 2 is a plot showing a sequence of potential contours at times numbered from 1 to 6 , following the collision history. Only a few contour lines around the extremal amplitudes are drawn for clarity, but at lower amplitudes some noise grows. Breaking and exchange of the dipole poles is produced at time labeled 2. This pairing of the positive and negative parts of the dipoles is followed by an oscillatory trajectory of each of the newly formed "dipoles," characteristic of biased dipoles [22]. The interaction process shown in Fig. 2 can be summarized by

$$
D_{+}+D_{-} \rightarrow D_{-1}+D_{-2}+\text { (radiation) }
$$

On can think that the differences observed are not really related to the value of $\Gamma$ but that they are inherent to the individual dipole parameters: very large dipoles break, small ones are more stable. In fact, the nature of the perturbation is very important (this is given by the collision time in the parameter $\Gamma$ ). To investigate this point we took the same dipoles as before, the marginal case $\Gamma \approx 1$, and performed a collision with an impact

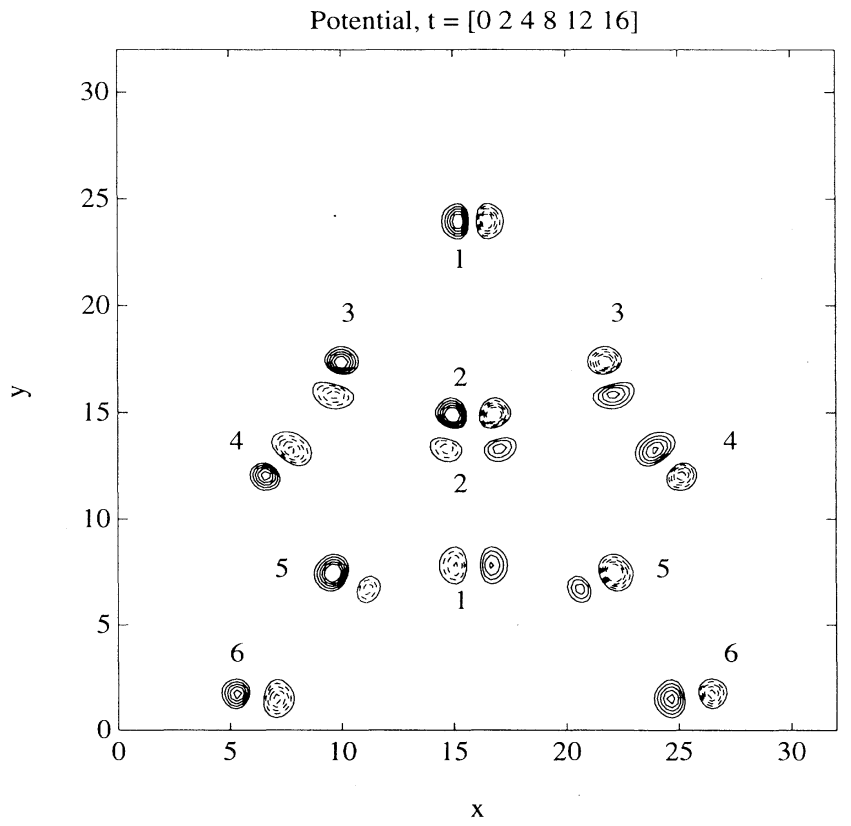

FIG. 2. Head-on collision of two dipoles in the Hasegawa-Mima case. Marginally fast collision case. A sequence of times labeled from 1 to 6 is shown. When the nonlinear time scale is of the order of the collision time scale, the dipole interaction is very complicated. Here by an exchange process two dipoles are formed. The dipole parameters are $v_{+}=3, r_{+}=1.44, v_{-}=-5, r_{-}=1.13$.

parameter different from zero. Obviously, the nonlinear time - intrinsic to dipoles - is not changed, but the collision time is reduced as the cross section diminished, giving a larger value of $\Gamma$. The result is shown in Fig. 3. The collision, as expected, is almost elastic,

$$
D_{+}+D_{-} \rightarrow D_{+}^{\prime}+D_{-}^{\prime} \text {. }
$$

Although some waves are produced, the two dipoles are practically not modified by the interaction. The same type of collision with an impact parameter the size of the dipole radius was studied by Horton [21]. However, in this case $\Gamma$ is in the inelastic regime. In contrast to Fig. 3, in the simulation by Horton, like regions of vorticity, which are directly in contact during the collision, merge to form an isolated monopole.

In conclusion to this discussion on the interactions of dipoles in the conservative (Hasegawa-Mima) case, we observe, taking dipoles as a paradigm of more general vortices, that nonlinear coherent structures are weakly perturbed by waves and create other structures by collision. We observed the formation of different types of coherent structures and in particular, the monopoles of Fig. 1. We remark that these monopoles are almost circular and that the relation of the vorticity $(\Delta \phi)$ and potential is linear inside the monopole, as can be seen in Fig. 4. This means that the Poisson bracket becomes small, and then the total potential can be split into two 


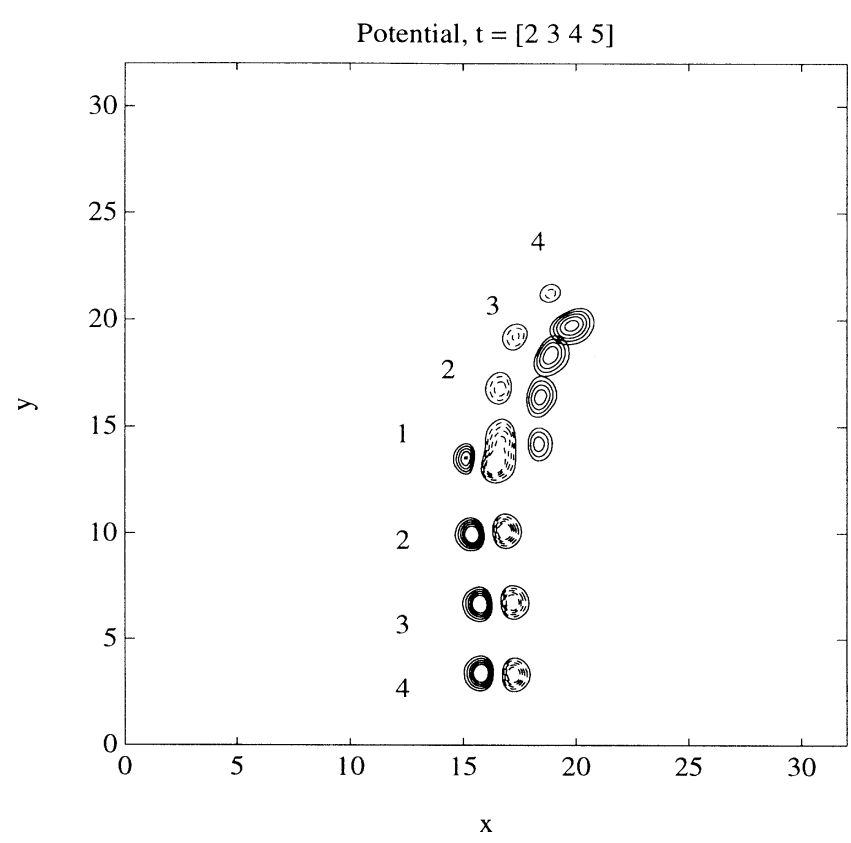

FIG. 3. Head-on collision of two dipoles as in Fig. 2 but with an impact parameter equal to the radius of one dipole.

components: the vortices and the waves, both kinds of objects interacting weakly.

Therefore, monopoles are close to the solution of the equation of freezing of the generalized vorticity $(\Omega=$ $\Delta \phi-\phi+x)$. As anticipated in the Introduction, nonlinear interaction can result in the formation of coherent structures, solutions of Eq. (1.1), rather than evolving as in an "inverse cascade" scenario, to a random state. These coherent structures can be considered in some sense as "particles," they can be characterized by only a few parameters like position and velocity and some internal degrees of freedom (amplitude, size). The reason for its robustness can be found in the fact that they interact only weakly with waves, because their velocity is generally different from the phase velocity of waves, then "resonant" interaction is negligible.

\section{B. Dipole collisions \\ in the unstable-dissipative case}

When nonadiabatic effects are taken into account, the linear dispersion relation deduced from Terry and Horton equation contains the growth rate (see Fig. 5),

$$
\gamma_{k}=\frac{\delta_{0}\left(k^{2}-c_{0}\right) k_{y}^{2}-\nu k^{4}\left(1+k^{2}\right)}{\left(1+k^{2}\right)^{2}+\delta_{0}^{2}\left(k^{2}-c_{0}\right)^{2} k_{y}^{2}} .
$$

For large values of $k$ the viscosity dissipation dominates, whereas for smaller values of $k$ the growth rate becomes very anisotropic. Its maximum, reached at intermediate values of $k_{y}$ and $k_{x} \approx 0$, is proportional to $\delta_{0}$, and a positive $c_{0}$ gives large-wavelength dissipation [20]. In Fig. 5 we plot the growth rate for typical parameters, showing its anisotropy at low wave numbers.

It is important to investigate the behavior of coherent structures, in our case the dipoles, when nonideal effects like instability in a range of modes and large $k$ dissipation are present. In order to study these effects we simulated the head-on collision of slow dipoles, using the same initial condition as in the Hasegawa-Mima case of Fig. 1. We considered two extreme cases, with small and large dissipation rate, both with a positive growth rate at intermediate $k$. At first glance, one can think that, for a given rate of instability, when dissipation is weak, dipoles would be destroyed. In fact, the actual behavior of dipoles is more complicated, the instability is a linear process that is more effective on linear drift waves than on nonlinear structures. We will see that the ability or inability of vortices to emit drift waves is essential to their stability.

\section{Small dissipation case}

The maximum growth rate is about $\gamma_{k} \approx 0.1$, the dissipation coefficient is $\nu=0.01$. The temporal evolution of the total energy shows that the system turns out to be globally dissipative, the energy is monotonically decreasing in time. Figure 6 shows the electric potential at different times. Qualitatively, the state after the collision
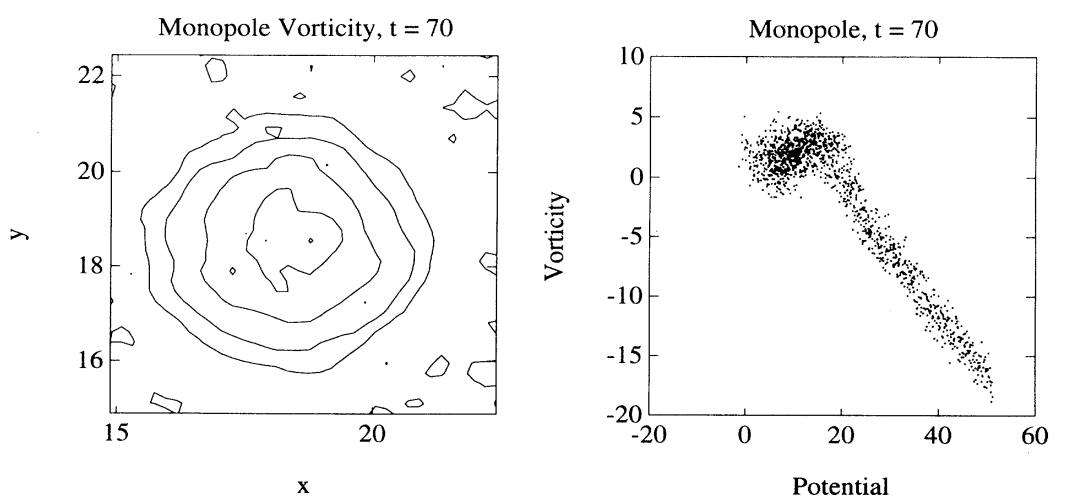

FIG. 4. Detail of the monopole of Fig. 1 (left), vorticity field, and (right), vorticity vs potential showing a strong correlation. 


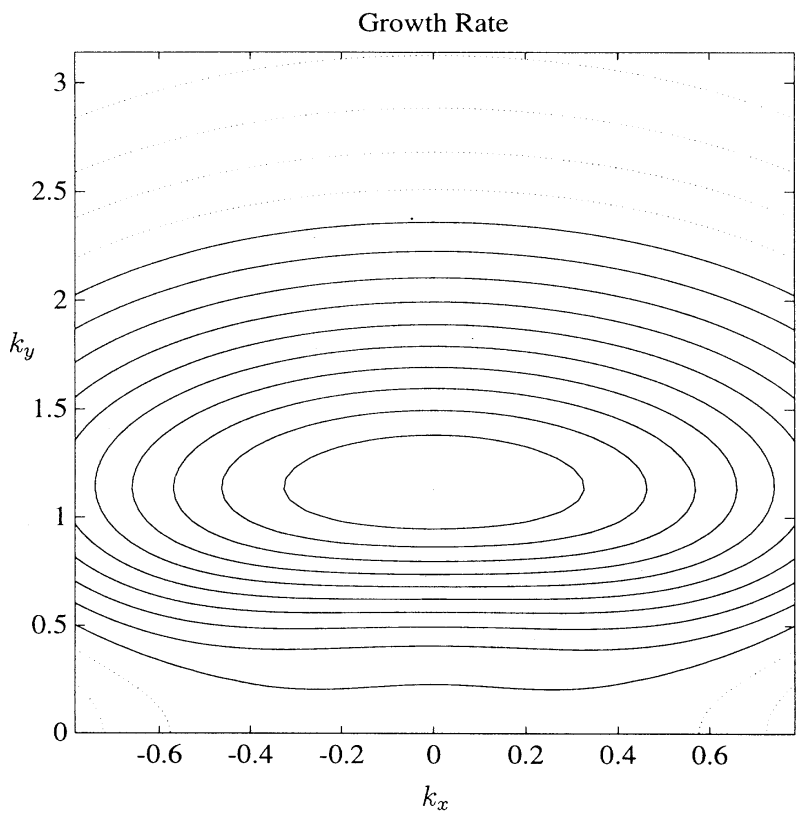

FIG. 5. Growth rate of the drift wave instability described by the Terry-Horton model, as a function of $k_{x}$ and $k_{y}$. Contour plot, the maximum is about 0.15 . The growth rate is anisotropic at low $k$ but becomes isotropic at larger $k$. Parameters are $\nu=0.15, \delta_{0}=1, c_{0}=0$.

is similar to the one for the ideal case: the positive velocity dipole survives after the collision, while the negative velocity one splits to form a pair of monopoles. However, two main differences with respect to the pure HasegawaMima case are worth noting. First, the trajectory of the vortices during the collision differs in that the positive velocity dipole passes directly inside the negative velocity one, while in the dissipationless case dipoles are momentarily broken and describe a large half circle before partially recombining. Second, the wake present in the ideal case is now less developed. The positive velocity dipole is progressively damped.

\section{Large dissipation case}

We will see that increasing the dissipation rate can result in destruction of the dipole. For the sake of comparison with the results in the small dissipation case, the maximum growth rate is of the same order of magnitude as before, the dissipation coefficient is instead one order of magnitude larger, $\nu=0.15$. One consequence of this change is that the maximum of the growth rate is slightly shifted to lower $k$. The temporal evolution of the total energy differs from the previous low dissipation case. Surprisingly the total energy, after an initial fall, increases and the system becomes unstable. We remark that, despite the change of parameters initially the dipole is only damped, and the system is (at less initially) globally stable. However, as shown in Fig. 7, the evolution of the system changes dramatically. The negative velocity dipole reverses its velocity in the first stages of the simulation, and the collision does not actually take place. Dipoles emit a large amount of drift waves. Both dipoles are destroyed, and more significantly, the wake of drift
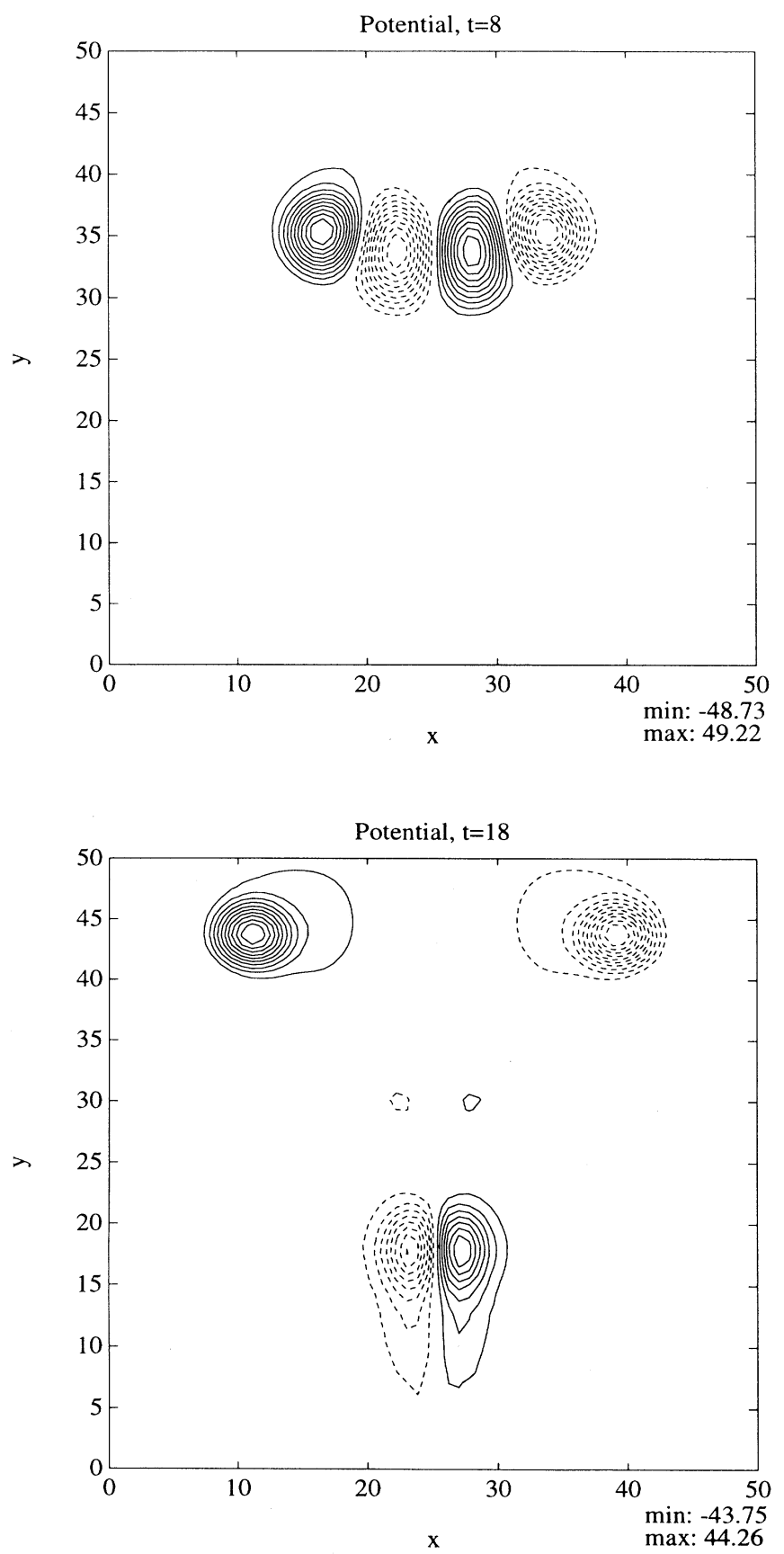

FIG. 6. Head-on collision of two dipoles in the Terry-Horton model. Low dissipation case. Contour plots at (top) $t=8$, the collision time, and (bottom) $t=18$. The final state is similar to the one in Fig. 1, but the trajectory of dipoles is different during the collision, no wake is visible. Parameters: $\delta_{0}=1, \nu=0.01$. 


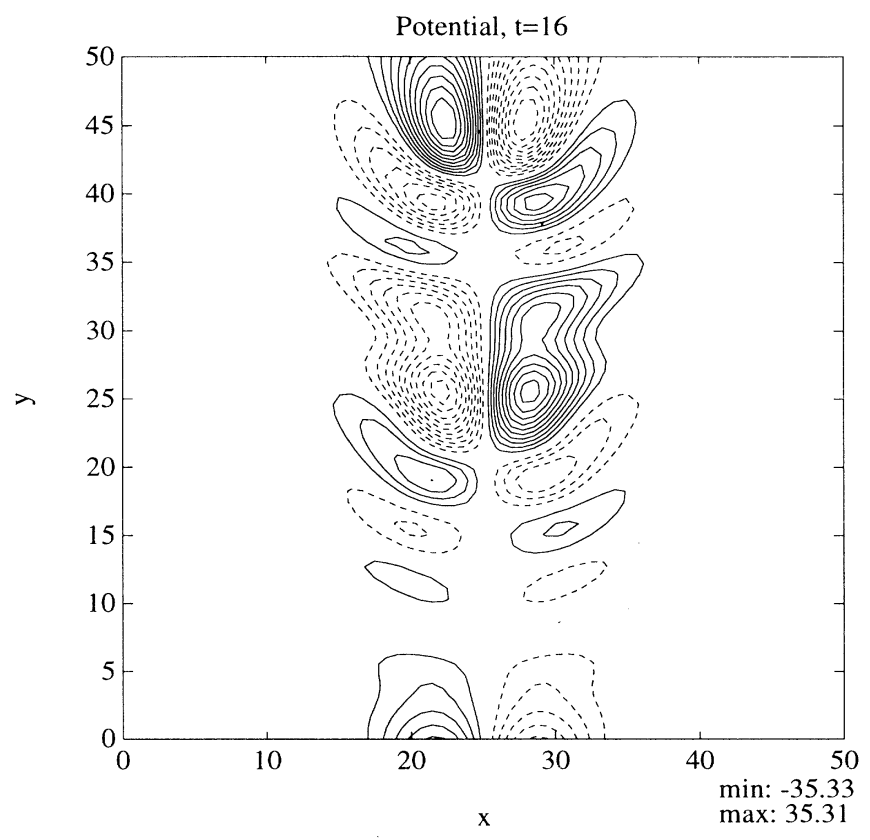

FIG. 7. Head-on collision of two dipoles in the Terry-Horton model. High dissipation case. The initial state is the same as in Fig. 6. Contour plot at $t=16$. Strong emission of unstable drift waves destroys the vortices. Parameters: $\delta_{0}=1, \nu=0.15$.

waves is sensitive to the instability of the system and starts to grow. The growth of the total energy may be explained by this instability of the wake.

These simulations illustrate clearly the influence of the wave field on the coherent structures. Due to a strong damping, the dipole is slowed, and then enters the region of wave propagation: the closer the velocity of the vortex to the phase velocity of waves, the stronger their interaction. The result of this interaction is the generation of a wake of unstable waves. This does not mean that longlived vortices cannot survive in this case; this depends on the actual parameters of the dipole (or other kinds of vortices). In fact, the long-time evolution of the system, as we will see in the sections devoted to the study of turbulence, tends to form relatively stable coherent structures.

\section{DECAYING TURBULENCE}

As mentioned in the Introduction, the Euler equation of $2 \mathrm{D}$ hydrodynamics and the Hasegawa-Mima equation have the same kind of nonlinearity, but they differ in linear properties. The Hasegawa-Mima equation possesses a real part of the frequency describing drift waves and an intrinsic spatial scale. In the case of pure decaying random initial conditions (with a Gaussian distribution and a broad spatial spectrum), the Euler system is known to evolve to a kind of thermodynamic equilibrium state $[26,27,38,39]$. One can naturally ask whether the same type of asymptotic state can be reached for the Hasegawa-Mima flow.

The Euler system evolution is characterized by an inverse cascade of the energy and the further development of coherent structures. Merging of these structures is pursued up to the formation of a pair of vortices filling the available space (the mean value of the vorticity is conserved). The asymptotic state, which is close to the sinh Poisson equation [27], can also be interpreted in terms of point vortices dynamics. Point vortices interacting with a logarithmic potential are a weak (exact) solution of the Euler equation. Equal sign vortices tend to form bounded systems, and then to accumulate, constituting a large unique structure, well separated from the opposite sign vortex [40]. Inverse energy cascade and formation of coherent structures are also well known for the Hasegawa-Mima flow [18].

However, although the Hasegawa-Mima equation may be written in the form of an equation for the conservation of a generalized vorticity $\Omega=\Delta \phi-\phi+x$ along stream (potential) lines [Eq. (1.4)], exact point vortex solution does not exist. Indeed, the last term in the generalized vorticity, which reflects the existence of waves, prevents strict localization (Dirac function) of this vorticity. Although some point vortex models were proposed [41-43] it must be emphasized that they are not an exact solution of the original equations, and more importantly, they do not preserve the motion integrals, and as a consequence they cannot describe the long-time behavior of the system.

One can loosely say that the Hasegawa-Mima equation not only has point vortex degrees of freedom (associated to the $\Delta \phi-\phi$ part of the generalized vorticity) but it also has supplementary degrees of freedom associated to waves ( $x$ term of $\Omega$ ). The long-time state of the system may be essentially different from the pure point vortex gas. Such a system of interacting point vortices and waves can have very complex and likely nonstationary asymptotic states [18]. On the other hand, the introduction of an intrinsic length scale (represented by the term $-\phi$ of $\Omega$ ) can also influence the thermodynamic properties of the system, in this case vortices interact with short-range forces (a modified Bessel function replaces the usual logarithm).

Moreover, by the interplay of linear and nonlinear mechanisms, the system dynamically may develop a characteristic (nonlinear) scale, basically the coherent structure size, independent of the box size, and then evolve to a state where coherent structures (associated to small values of the Poisson bracket) and waves coexist; the interactions between the two objects being weak, the system attains a quasistationary state.

To investigate the long-time behavior of the HasegawaMima flow we performed a series of extensive numerical simulations with various initial conditions (random phased potential with power-law spectrum), box sizes, and dissipation coefficient: (i) Box size $32^{2}$, dissipation coefficient $\nu=10^{-2}$, potential spectrum $\left|\phi_{k}\right|^{2} \approx k^{-10}$ (corresponding to a $k^{-7}$ energy spectrum), simulation time $t=500$. (ii) The same as (i) but with a box size of $64^{2}$ to allow testing of the influence of the box in the 
system's evolution, $t=700$. (iii) Box size $64^{2}$, smaller dissipation $\left(\nu=10^{-4}\right)$ to compare with (ii) and test the influence of viscosity, the potential spectrum is as before $\left|\phi_{k}\right|^{2} \approx k^{-10}, t=500$. (iv) The same as (iii) but with an initial spectrum richer in small scales: $\left|\phi_{k}\right|^{2} \approx k^{-6}$, close to the inverse cascade spectrum, $t=800$.

Box sizes are large enough to avoid the influence of boundary conditions (the linear scale is 1 ), and as the mesh size is $256^{2}$, we have reasonable numerical precision on small scales. The simulation times (up to 800 periods) were chosen to obtain an almost stationary state, not only for the statistical "macroscopic" properties but also for the spatial structure. The slight dissipation used allows the total energy to be practically conserved during the system evolution. Some of the results are presented in the form of contour plots of the potential $(\phi)$ and vorticity $(\Delta \phi)$ fields. Solid lines are positive values and dashed lines represent negative values. The maximum and minimum of the potential are written at the bottom of the figures. The time is generally specified in the title of the figure.

\section{A. Case (i): Small box simulation}

The system evolves by successive merging steps up to a state where only two vorticity structures of opposite sign survive. In Fig. 8 we show contour plots of the potential and the vorticity at time $t=500$. Although at first sight it seems that the situation is similar to the Euler $2 \mathrm{D}$ system, the vorticity field is very complicated, and it is not possible to say that the system reached any kind of "thermodynamic" equilibrium state. Indeed, the vorticity field is evolving in time, interactions and deformation of the structures are observed, and the spectrum of scales is broad with a random component. This is in contrast to the Euler situation where the vorticity field reaches an almost static and ordered state. The last generation of merging processes occurred at $t \approx 300$, then for 200 periods (turnover time of an eddy of size and amplitude unity) the vortices were convected and deformed by the flow but conserved, and no tendency to an ordered, static, configuration appeared. The lifetime of these structures is much larger than its own turnover time by at least a factor 100. It is also important to note that the size of the monopolar structure is smaller than the box size, having a radius of about 5 length units. Therefore even for this modest box size the field can be characterized by a superposition of coherent structures and waves interacting weakly. We remark that the phase velocity of drift waves with wavelength comparable to the vortex size is about 0.4 smaller than the monopole velocity, which is about 1 .

\section{B. Case (ii): Large box simulation}

The ability of the system to create a definite nonlinear scale is essential to its long-time behavior independent of the box size. Some evidence of this was given in case (i), where the generated coherent structures were smaller than the box size. However, in this case only two structures remained, and then we can ask whether the number and size of the coherent structures were independent of the box. To investigate this point we performed a second
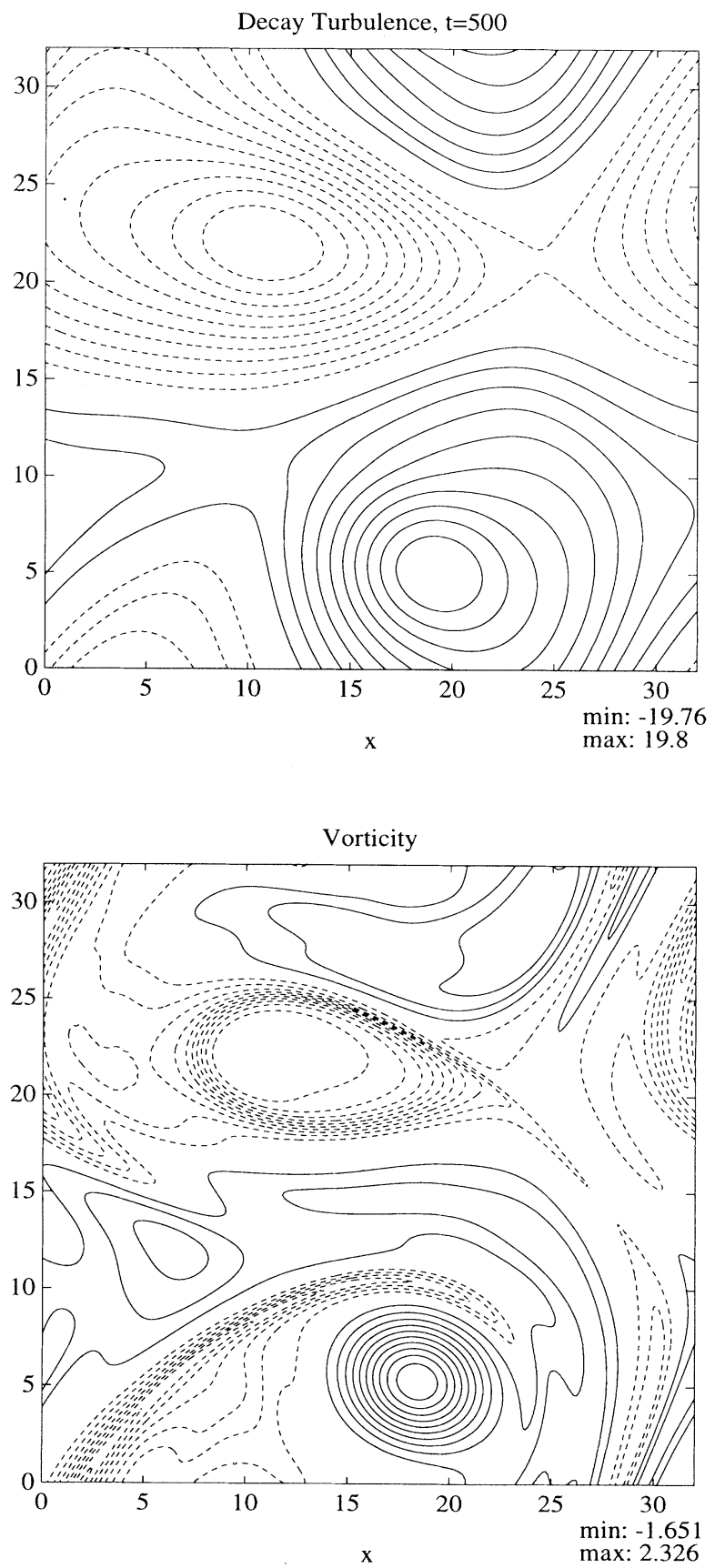

FIG. 8. Decaying turbulence simulation, case (i). Potential (left) and vorticity (right) contour plots at time $t=500$. Merging of the last vortices occurred at $t \approx 300$. For more than 160 turnover times of the largest coherent structure, the number of vortices and the global spatial pattern remain unchanged. We see that potential and vorticity are well correlated (cf. Fig. 4). The coherent structure size is about five length units. 
simulation of decaying turbulence with the same parameters as in (i) but with a box of $64^{2}$ area. The initial stages of the evolution are dominated by the increase of the vortex size, through various generations of the merging process. As a result a few coherent structures, monopole and dipole vortices, are formed. The lifetime of these structures is extremely large. The fundamental observation is that the size of the resulting vortices is of the same order as in the smaller box simulation. This fact supports the idea of a nonlinear selection of the vortex size, independent of the boundary conditions.

Figure 9 shows the potential (left) and the vorticity (right) at $t=480$. The number size and form of the structures are almost the same at the two times. Essentially monopolar vortices are present, notably the one at the bottom of the plots with an amplitude of -28 . All these structures are traveling in the positive direction (from the left to the right of the figures). In Fig. 9 we observe a definite correlation between potential and vorticity, a characteristic property of coherent structures. The state of the system is roughly stationary; no qualitative changes are observed up to time $t=700$. The number and the shape of the larger structures are conserved during this time. We see that a few isolated structures are present in the field, with a size of 5 , as in case (i), much smaller than the box length 64 . Therefore, cases (i) and (ii) show the appearance of long-lived coherent structures with a characteristic size independent of boundary conditions. These structures are of the same type as the monopole of Fig. 4. This result was obtained with a relative high value of the viscosity; we now want to test this hypothesis in the case of low dissipation, when small scales can almost freely develop in the evolution of the system.

One important consequence of the large dissipation rate is that the enstrophy is not conserved: the distribution of the vorticity can evolve from the initial Gaussian to some other distribution. The appearance of stretched vorticity gradients is a signature of non-Gaussian statistics. Indeed, in Fig. 10 we show the histograms of the vorticity and one component of the vorticity gradient (both components have the same statistics), which deviates significantly from the normal distribution. In the next paragraph we study the low dissipation rate case, for which enstrophy is almost conserved.

\section{Case (iii): Low dissipation}

The parameters of the simulation were chosen to validate the results of case (ii) when a very small dissipation is present $\left(\nu=10^{-4}\right)$; this low value of the viscosity coefficient implies damping of only a few large $k$ modes, comparable with the Fourier cutoff length - related to space discretization. Other parameters are similar to case (ii).

The first important result is that, despite the fact that initially no small scales are present (a $k^{-10}$ initial spectrum was adopted), these small scales are dynamically created, supporting the existence of some direct cascade mechanism in addition to the usual inverse energy cascade paradigm. The second observation is that the general large-scale behavior of the system is unchanged with
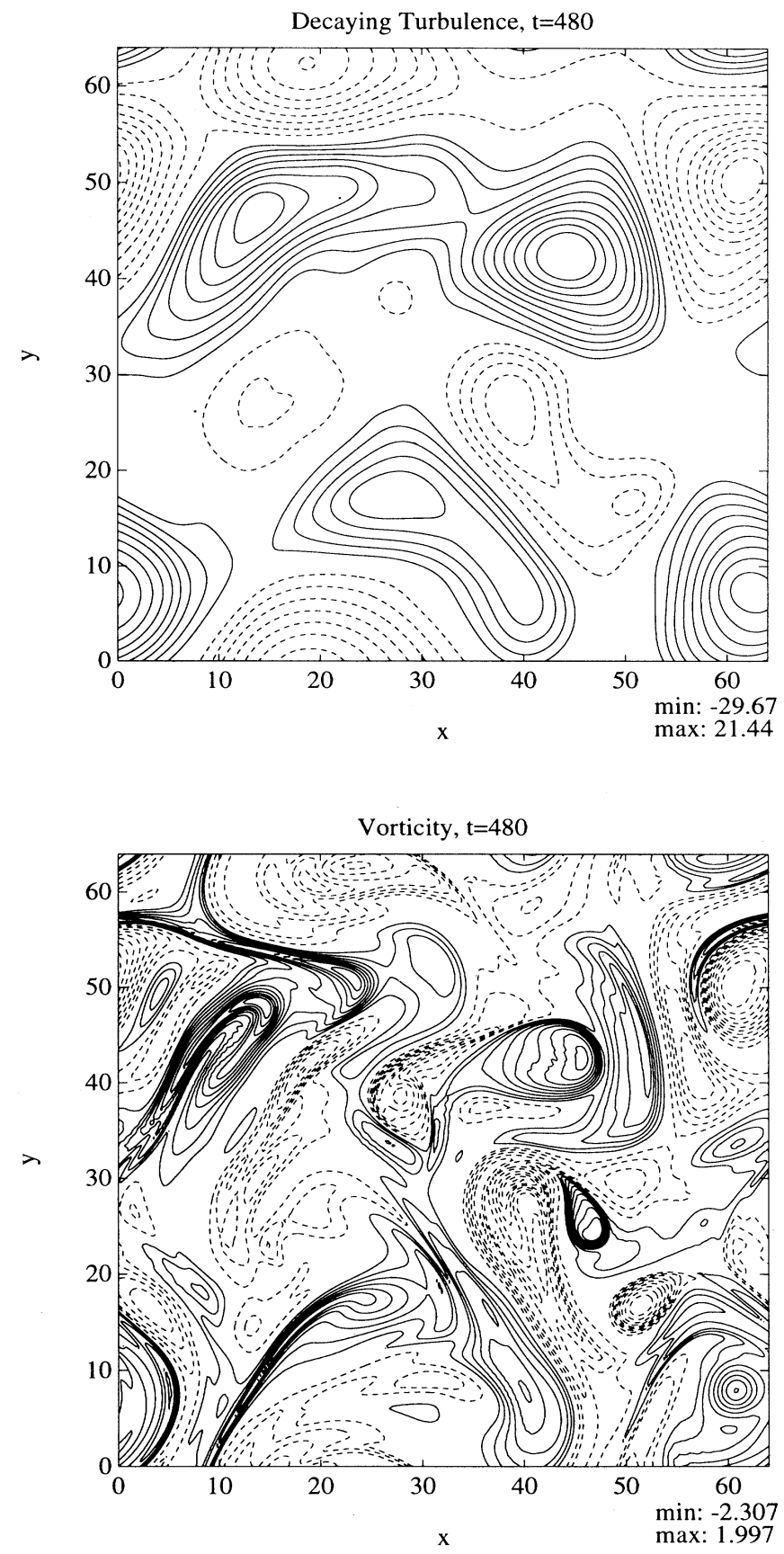

FIG. 9. Decaying turbulence simulation, case (ii). Potential (left) and vorticity (right) contour plots at time $t=480$. Coherent structures of the same type as in Fig. 8 are present. Notably their size is of the same order of magnitude, showing that it is independent to the box size. Although the potential shows a very simple pattern, the vorticity shows a rich variety of small scales and elongated gradients. 
respect to the previous case: formation of coherent structures approaching an approximate stationary state; the size of the structures is always of about 5 at comparable times (Fig. 11). Third, the small-scale activity changes the statistical properties of the flow. In the regime where small scales are damped, and the flow is completely dominated by the coherent structures, practically unperturbed, the distribution probability of the
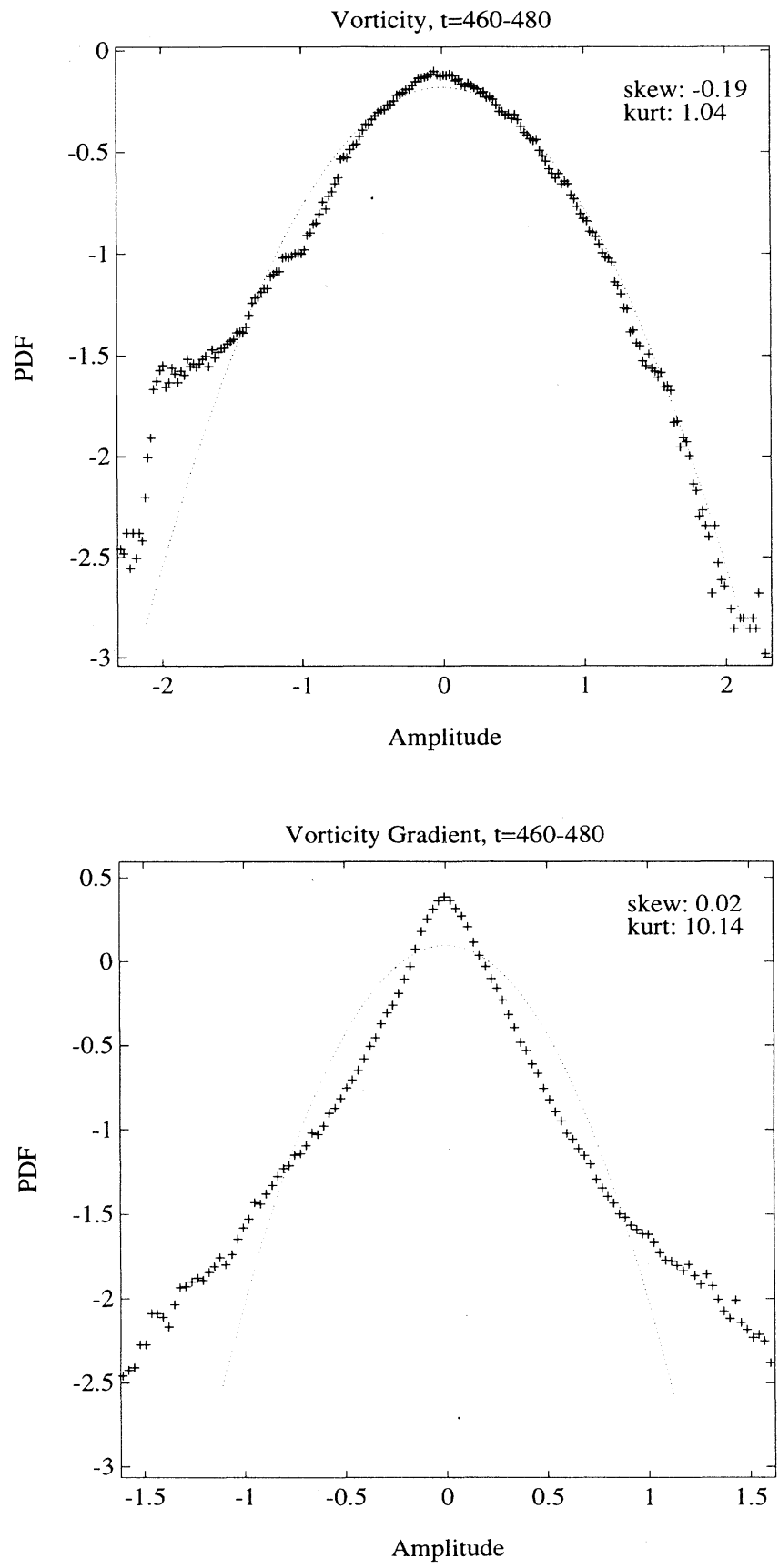

FIG. 10. Histograms of the vorticity (top) and one component of its gradient (bottom). For comparison we show the Gaussian distribution, appearing as an inverted parabola (dotted line), and the values of the skewness and kurtosis.

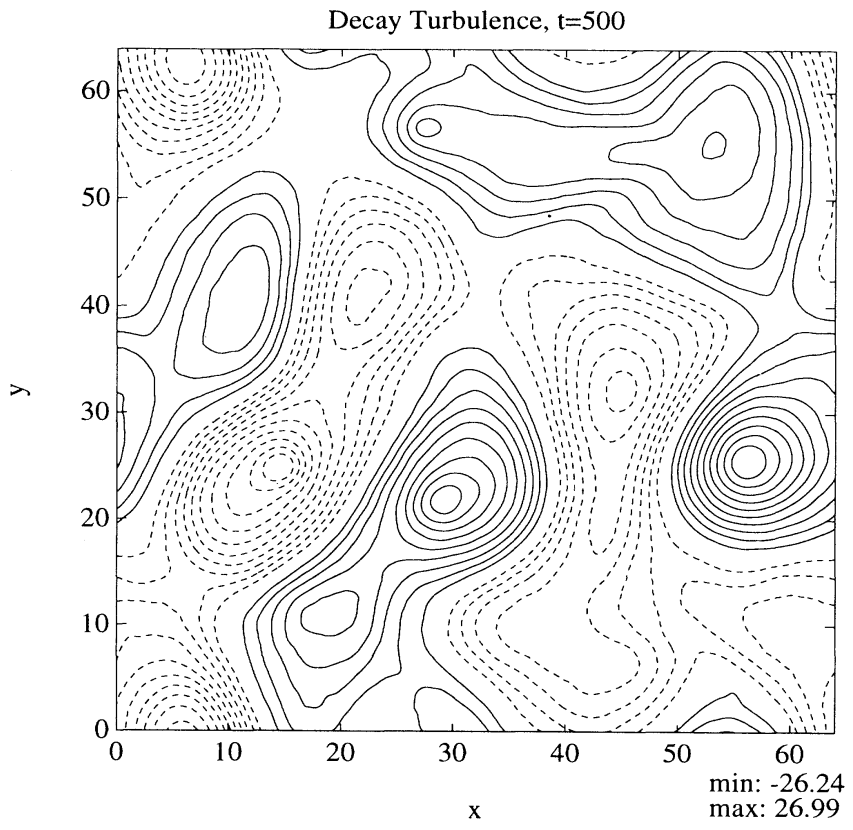

FIG. 11. Decaying turbulence simulation, case (iii). Potential contour plot at $t=500$. In this case viscosity is very low and then small scales are enhanced. Although large structures are present, small scales dominate the vorticity field, giving an almost Gaussian distribution.

vorticity is very far from being Gaussian, large amplitudes are too "frequent," the gradients are concentrated between rather flat vorticity profiles. Diminishing the damping allows small scales to develop and, notably, the vorticity histogram becomes Gaussian.

In contrast, the probability distribution of the potential amplitudes, less sensible to small scales than vorticity, remains non-Gaussian. It is worth noticing that without dissipation the initial vorticity distribution (in our case Gaussian) must be conserved by the flow, not the potential distribution. This is a manifestation of the infinity of invariants of the Hasegawa-Mima equation.

Therefore, we can conclude from these results, and the ones presented in the previous paragraph, that the dissipation rate influences the statistical properties of decaying turbulence, but the behavior of large-scale vortices remains the same (lifetime and size of the largest structures is equivalent in both cases). In the high dissipation rate regime significant intermittency is observed (in the sense that probability distribution functions of the fields are essentially non-Gaussian). Moreover, when the dissipation rate is negligible, conservation of generalized vorticity guarantees the Gaussianity of the fields (even higher moments of the vorticity remain Gaussian in this simulation). The study of the statistical properties of the coherent structures and the background of small fluctuations is the issue of the following paragraphs. 


\section{Case (iv): Low dissipation and large spectrum}

In the last case we investigate the influence of the initial condition on the evolution of the system. We released the restriction of an initial large-scale field and simulated the evolution of a broad $k$ spectrum. We also take a slightly smaller initial amplitude and a viscosity of $10^{-4}$. Figure 12 shows a sequence of the potential between $t=500$ and 800 .

Following the potential evolution from $t=500$, where there is a prominent positive monopole, we see that this monopole slowly associates to another (negative) monopole to form a dipolar structure: polarity is in correspondence with the direction of motion; the amplitudes of the poles are progressively balanced. During this time, the double-peaked negative structure tends to split into two parts: one merges to the positive monopole to form the dipole, the second one forms an isolated monopole. This splitting process is achieved at $t=650$.

We note that also in this case the overall pattern - the large-scale features - are similar to other regimes. At the final simulation time $t=800$, the potential is dominated by essentially two coherent structures: a monopole and a dipole. These two vortices are present for about 300 periods. Both are moving in the positive- $y$ direction, at a velocity of the order of the drift velocity. The dipole velocity is slightly larger than the monopole velocity, which is within the error, equal to the drift phase velocity.

Observation of the breaking of a large structure into distinct coherent structures is fundamental to the understanding of the quasistationary regime attained by the evolution of a random initial condition. Indeed, we mentioned that the system stay for very long periods in a state characterized by the presence of large vortices and a field of waves interacting weakly. Although initially the system evolves through successive generation of growing vortices, very large vortices tends to break out. These two competing mechanisms are responsible for the observed "saturated" state.

\section{E. Spectrum and proper orthogonal decomposition}

One indication that the system reaches a quasistationary state is the persistence of its statistical properties, such the Fourier power spectrum. In Fig. 13 we show the potential spectrum $W_{k_{x}}$ and $W_{k_{y}}$, integrated over $k_{y}$ and $k_{x}$, respectively, at times 500 and 800 . We observe that the overall form and the value of the slopes at intermediate and large $k$ are practically the same.

We can also measure the spatial coherence of the potential using the proper orthogonal decomposition (POD) [29-31]. This consists in projecting the field

$$
\phi(x, y)=\sum_{n=1}^{M} A_{n} X_{n}(x) Y_{n}(y)
$$

where $X$ and $Y$ are orthogonal eigenfunctions of the correlation functions,

$$
C_{x}=\int d y d \xi \phi(\xi+x, y) \phi(\xi, y)
$$

and

$$
C_{y}=\int d x d \eta \phi(x, \eta+y) \phi(x, \eta)
$$

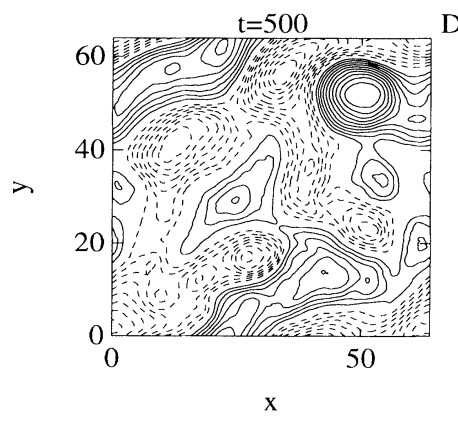

Deacaying Turbulence

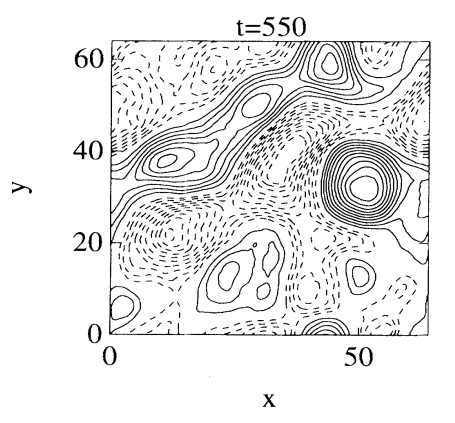

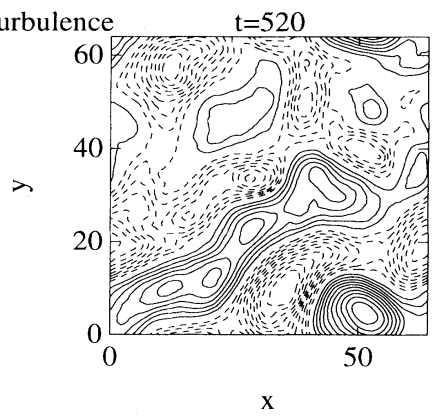

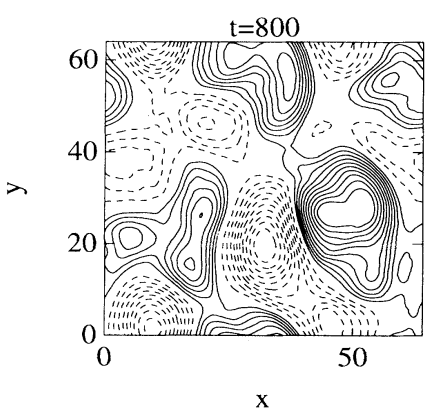

FIG. 12. Decaying turbulence simulation, case (iv). Sequence of the potential contour plots at times $t=500,520,550$, and 800 . We remark on the high stability of structures, whose liftime is of hundreds of turnover times. The "dipole" of the last figure appeared at $t \approx 650$. The drift velocity of structures in the positive $y$ direction is about 1 . 
respectively. In practice, we consider the field $\phi(x, y)$ as a matrix $\Phi=\phi_{x, y}$ where the indices span the computational mesh, and form the correlation matrices $\Phi \Phi^{T}$ and $\Phi^{T} \Phi$ whose eigenvectors are $X$ and $Y$, respectively (i.e., $\Phi \Phi^{T} X=A_{n}^{2} X$ and similarly for $Y$ ). This technique is also known as the singular value decomposition: $\Phi=X S Y^{T}$, where $S$ is a diagonal matrix containing the eigenvalues $A$. The weights $A_{n}$ are sorted in decreasing order, such that high correlated structures are related to
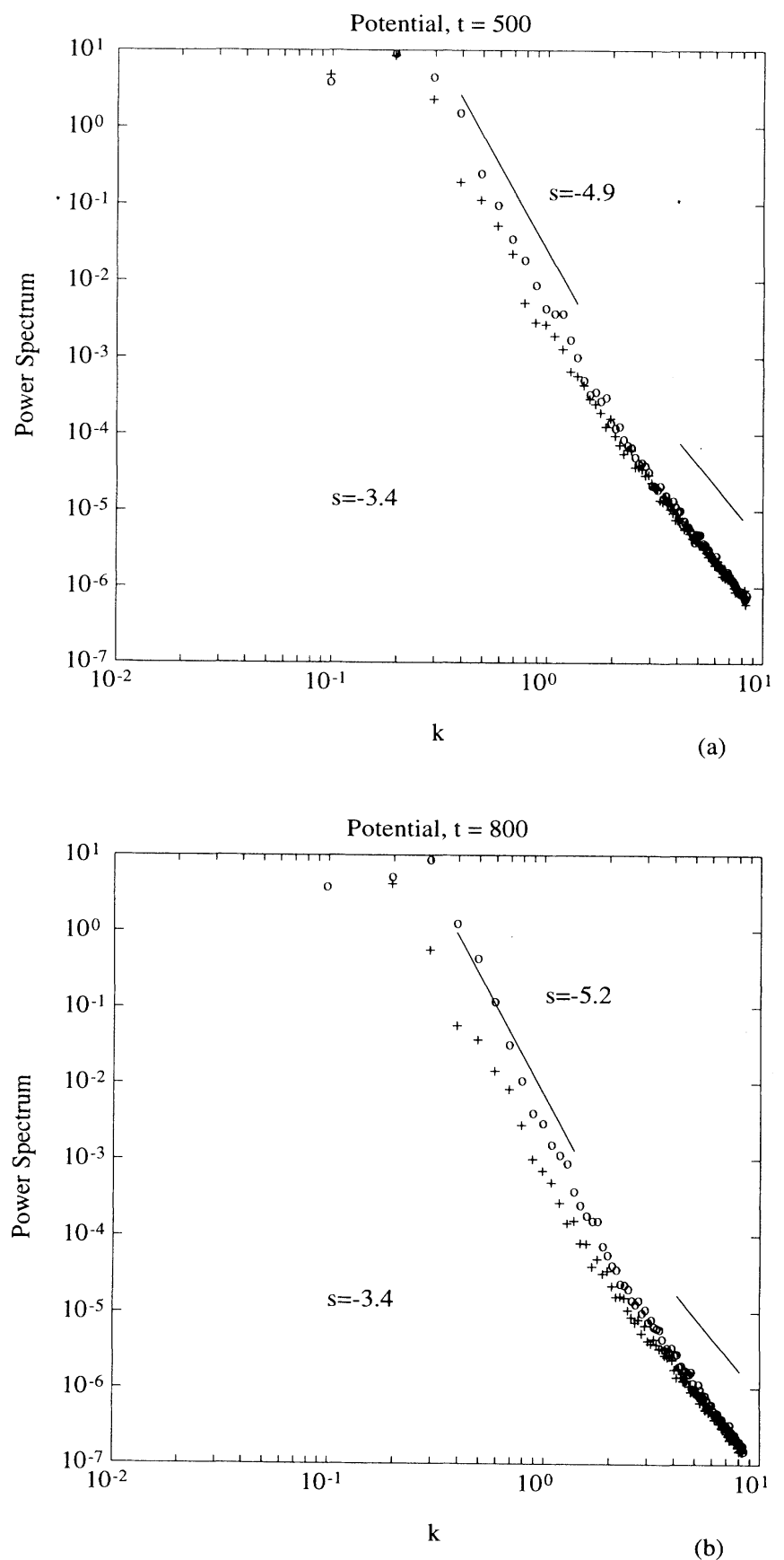

FIG. 13. Power spectrum of the potential at $t=500$ and $800\left(W_{k_{x, y}}=\sum_{k_{y, x}}\left|\phi_{\mathbf{k}}\right|^{2}\right)$. The slopes of the spectrum are indicated at intermediate and large $k$. the first terms in the decomposition. For a random field the POD spectrum follows the Fourier spectrum, but for correlated fields the eigenfunctions can be very different from Fourier modes, and in particular they can have a large Fourier spectrum, indicating that different scales are correlated. These properties of the POD are used to detect the correlated part of the signal (here the coherent structures) and to separate this coherent component to the noisy fluctuations. In Fig. 14 we represented the distribution of the weights $A_{n}$, for the electrostatic potential at $t=800$ (see Fig. 12). The distribution of $A_{n}$ shows that most of the information is concentrated in a few POD components. This statement is confirmed by the reconstruction of the signal using only the first four modes $(M=4)$ of the POD (Fig. 15).

The POD allows us to separate the contribution of coherent structures to the (spatial) power spectrum. Indeed, the Fourier spectrum of the reconstructed field has a slope of about -5 . Therefore, we can associate the steep part of the spectrum to the large-scale part of the potential. Moreover, neglecting the small-scale fluctuations, we find that the dynamics of the system can be described by only a few independent degrees of freedom, given by the relevant eigenfunctions of the POD. Moreover, the fact that the system may be described as consisting of two components, having different statistical properties, does not allow a simple interpretation of the power spectra found. In some sense this means that nonuniversal behavior is present, as in the cascade interpretation of $2 \mathrm{D}$ turbulence.

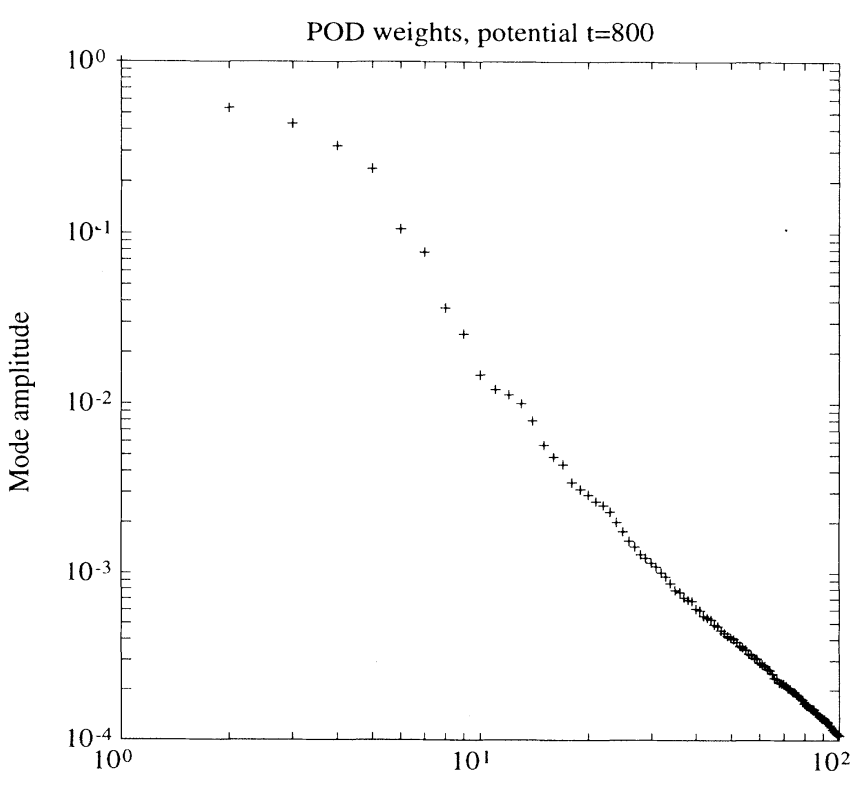

Mode number

FIG. 14. Distribution of weights of the proper orthogonal decomposition of the electrostatic field at $t=800$ of Fig. 12 . 


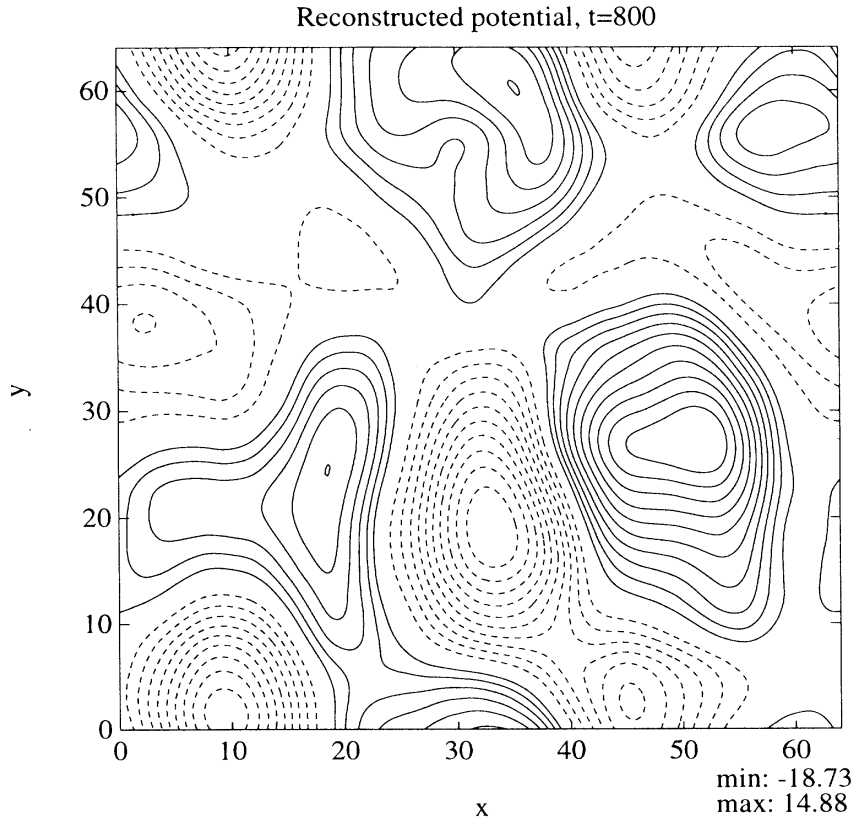

FIG. 15. Reconstruction of the field at $t=800$ of Fig. 12 using the first four modes of the proper orthogonal decomposition.

\section{DRIVEN TURBULENCE}

We also studied turbulence in the frame of the TerryHorton model. Although several sets of parameter values and choices of the initial wave spectrum were studied, we present here a simulation with $\delta_{0}=1, \nu=0.15$, and $c_{0}=0$, the computational mesh has $256^{2}$ points, the system is in a periodic square box of size 64 (spatial units), and the time step is $\Delta t=210^{-3}$. The simulation lasted for 200 periods. In order to test the ability of the system to reach a stationary state we put $c_{0}=0$, then no low- $k$ dissipation is included. Under these conditions, if only the inverse cascade of the energy operates, no stationary state can be attained, since any dissipation of the energy may compensate its accumulation at large scales.

Starting with small-amplitude random phased waves, the system evolves through an inverse cascade forming large-scales structures. After a rapid growth of the energy due to the linear instability, in spite of the absence of dissipation at large scales, a stationary state is established. This state is characterized by the competition of merging like-signed monopoles and the simultaneous development of secondary instabilities of large vortices which break down, a mechanism that we also observed in the pure decay case. Many of the elementary processes (fusion and breaking of monopoles, vorticity concentration) are in fact similar to the decaying case; the main difference is in global properties: a stationary regime with a well-developed power spectrum and stable probability distributions is established.

Long-lived monopoles are observed. Their velocity is generally in the forbidden dipole velocity range $(0<v<$ $\left.v_{d}=1\right)$ as in the decaying turbulence case. In Fig. 16 we show the potential field at $t=200$ and the (generalized) vorticity, defined as $L \phi[L$ is the operator given by (1.3), we call it TH (Terry-Horton) vorticity in the plots]; correlation between these two quantities means that the nonlinearity in Eq. (1.2) becomes small. This phenomenon, called nonlinearity depletion, might be re-
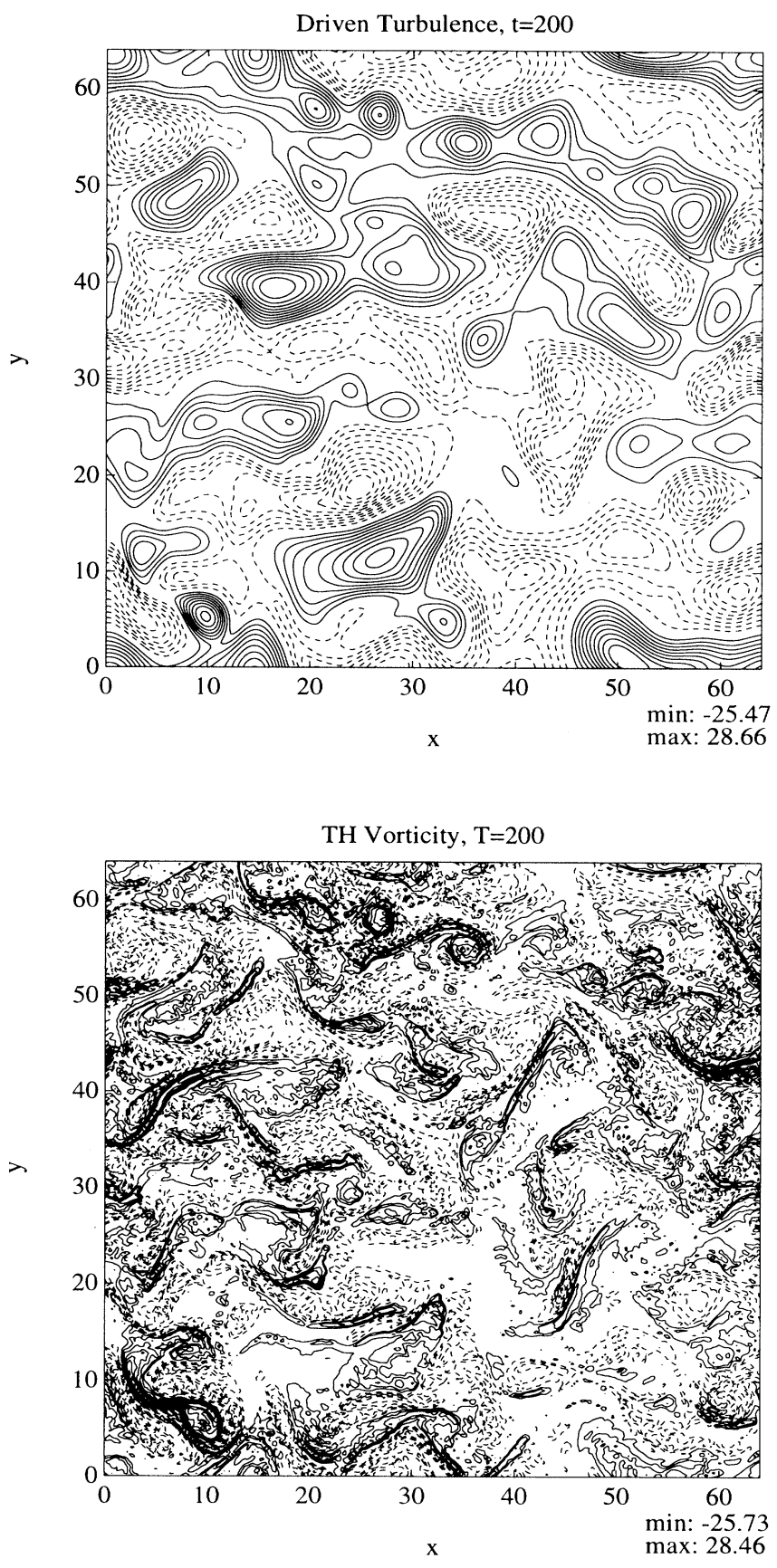

FIG. 16. Driven turbulence simulation (Terry-Horton model). Potential and (generalized) vorticity contour plots at $t=200$, in the stationary state. The liftime of individual vortices is about ten periods. 
sponsible of the saturation and stopping of the inverse cascade.

In order to test this idea, we perform a conditional average analysis of the potential and of the (generalized) vorticity [32]. This consists in sampling the field in such a way that a condition is satisfied, here we take only the
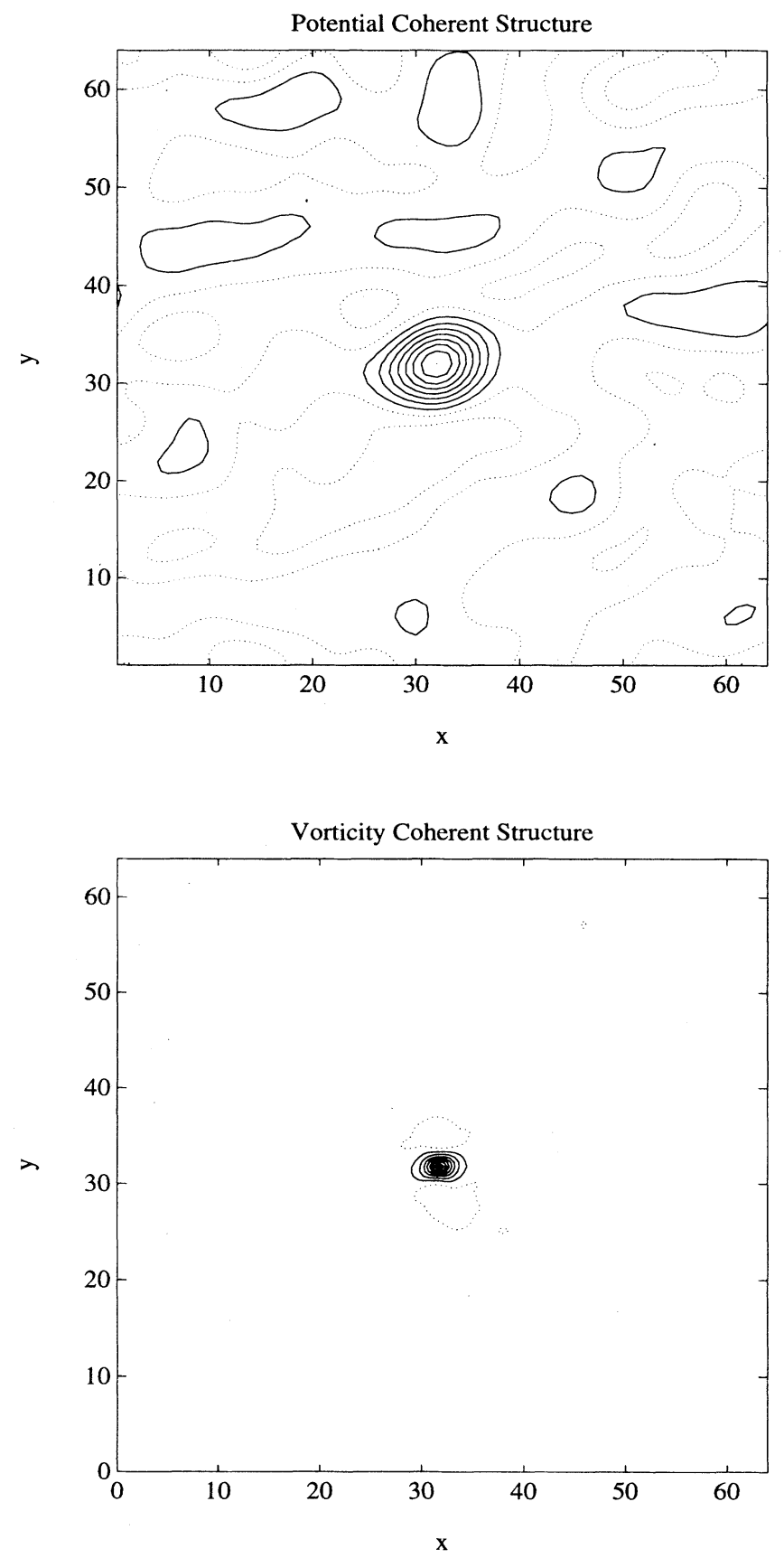

FIG. 17. Conditional average of the potential in the stationary state of driven turbulence. The resulting structure represents the typical form of a vortex (top). Conditional average of the (generalized) vorticity in the stationary state of driven turbulence. Incoherent fluctuations of the field are smeared out, and only the typical structure survives (bottom). subset of (grid) points for which the potential (or the vorticity) is larger than a given amplitude. For each point in the subset we shift the field in order to put the point at the center of the mesh (we take advantage of the periodic boundary conditions), in this way we obtain an image of the field around the points of amplitude larger than the threshold. We sum up all these images. We perform the average in the stationary regime, taking the potential at different times (separated by intervals of 10 periods to avoid spurious correlations). In this way the fluctuating part of the signal is smeared out and the typical form of the coherent structure emerges. These averages are shown in Fig. 17. We see that the vorticity field, which is very complex (cf. Fig. 16), gives a coherent structure, well localized as for the potential, and remarkably sharp with respect to the background fluctuations, which completely disappeared after the averaging. In Fig. 18 we show the scatter plot of the (generalized) vorticity versus the potential. The functional relation between these two quantities supports the assumption of the formation of coherent structures, and the consequent depletion of the nonlinearity. We stress the fact that, even if one takes the instantaneous $\phi$ and $L \phi$ of a particular vortex, one finds a definite relation, but more noisy due to the random contributions. This is related to the strong correlation between the peaks of $\phi$ and $L \phi$, which almost coincide in strong vortices.

The potential power spectrum is steeper than the pure inverse cascade spectrum, as can be seen in Fig. 19, its slope is $\approx-7.5$. We may think that this difference is due to the contribution of coherent structures. As in the decaying turbulence case, we do not expect here a universal cascade spectrum: the coherent structures introduce

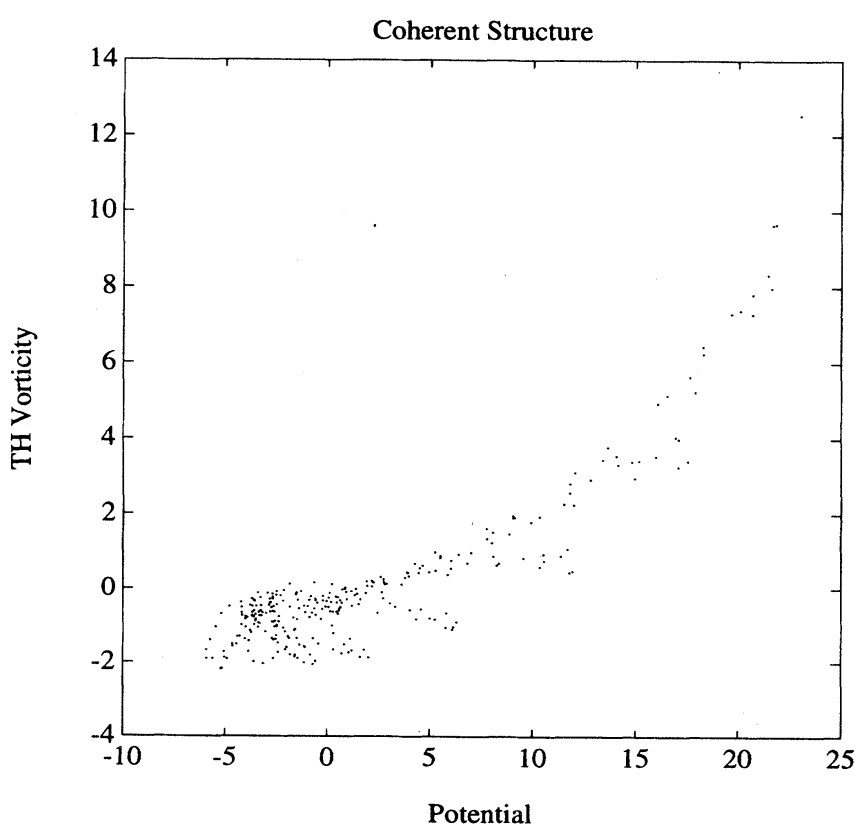

FIG. 18. Scatter plot of the (generalized) vorticity as a function of the potential, for the coherent structure obtained by conditional averaging. 


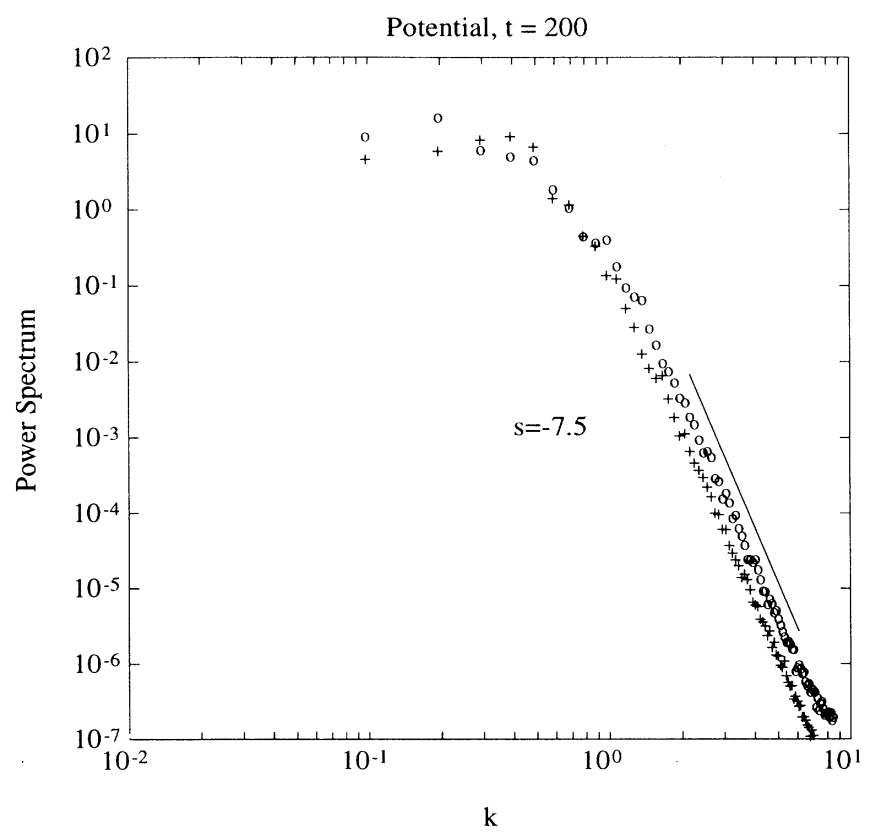

FIG. 19. Power spectrum of the potential for the driven turbulence at $t=200$. The slope at intermediate $k$ is -7.5 . The corresponding energy slope is -4.5 , much larger than the inverse cascade one. Symbols are as in Fig. 13.

nonlocal interactions in Fourier space. We also note that even at large $k$ we do not observe an exponential but a sharp algebraic decrease, although the dissipation term becomes important for moderate values of $k \approx 2$. We indicate that we do not use a hyperviscosity term, as used in other simulations. The separation of the contributions

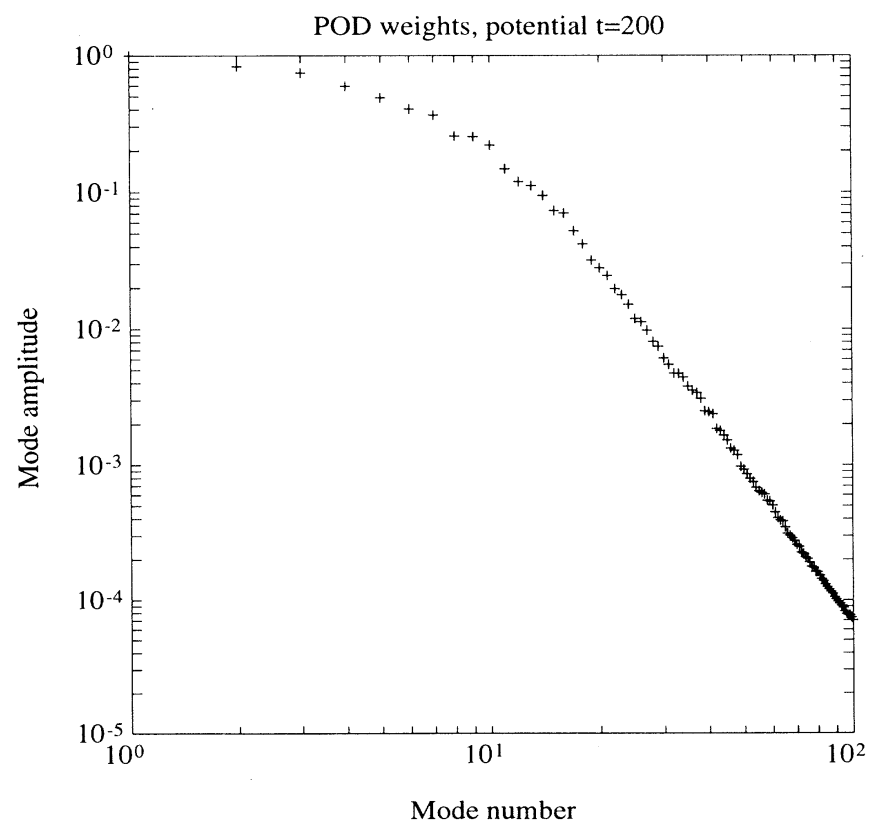

FIG. 20. Distribution of weights of the proper orthogonal decomposition of the potential at $t=200$ of Fig. 16 .

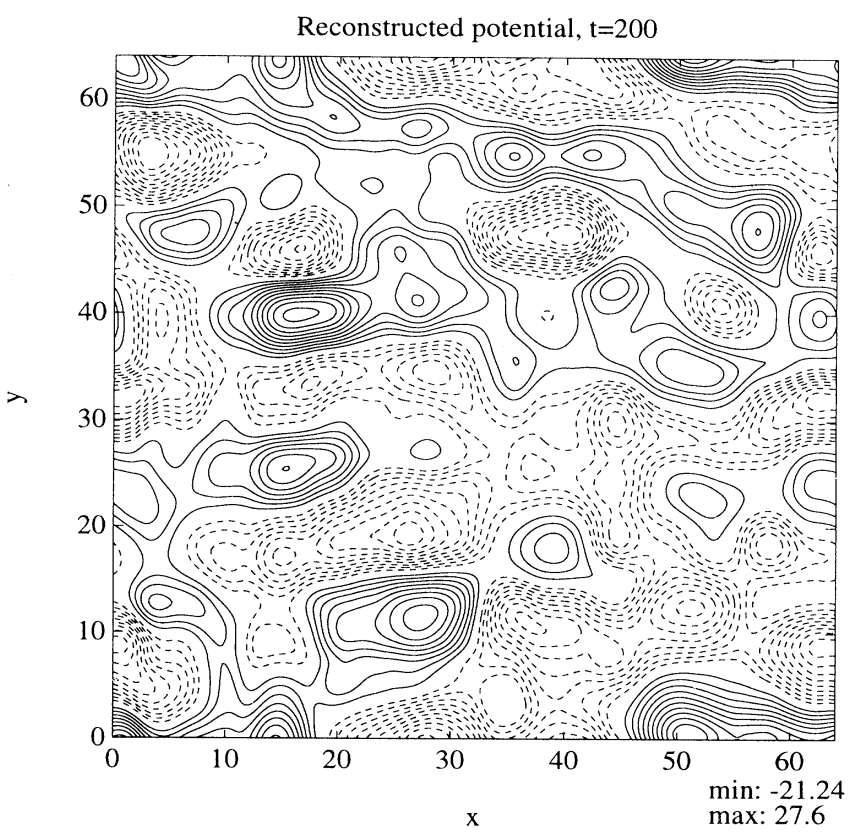

FIG. 21. Reconstruction of the field at $t=200$ of Fig. 16 using the first five modes of the proper orthogonal decomposition. Essentially all the large scale features of the field are correctly reproduced.

of coherent structures and random fluctuations can be done by the POD, as we did in the decaying turbulence case.

We show in Figs. 20, 21, and 22 the results of the POD applied to the potential field at time $t=200$. The distribution of weights is flatter (for the low modes) than in the case of decaying turbulence, indicating that more components are contributing to the field. However, to reconstruct the larger scales it is enough to keep only $M=5$ modes of the POD. The Fourier spectrum of the reconstructed field is almost the same for $M=5$ and $M=15$. We find a very steep slope at high $k(\approx-7.8)$, which is close to the total field power spectrum. This subset of the power spectrum corresponds to the contribution of large-scale (coherent) structures, which are well localized. We note that the POD eigenfunctions are far from being Fourier modes, the spectrum of the superposition of the five first modes being wide and decaying algebraically. Moreover, the random fluctuations, absent in these modes, must contribute to the power spectrum with a smaller slope, closer to the cascade spectrum.

This description is confirmed by the Fourier power spectrum of the difference between the actual potential at $t=200$ and the reconstructed potential using the first $M=15$ modes, Fig. 22 (the result is almost the same for $M$ as low as 5). This difference contains only the random part of the signal, in the sense of the POD. We note that the slope of the spectrum $(\approx-6.2)$ is consistent to the usual (inverse) cascade energy spectrum (at high $k$ we must multiply the potential spectrum by $k^{3}$ to obtain the energy spectrum). We also remark that at low 
$k$ small scales do not contribute very much, and it has a maximum corresponding to the maximum of the growth rate.

There is another interesting consequence of the presence of coherent structures in the stationary state of driven turbulence, the appearance of intermittency, in
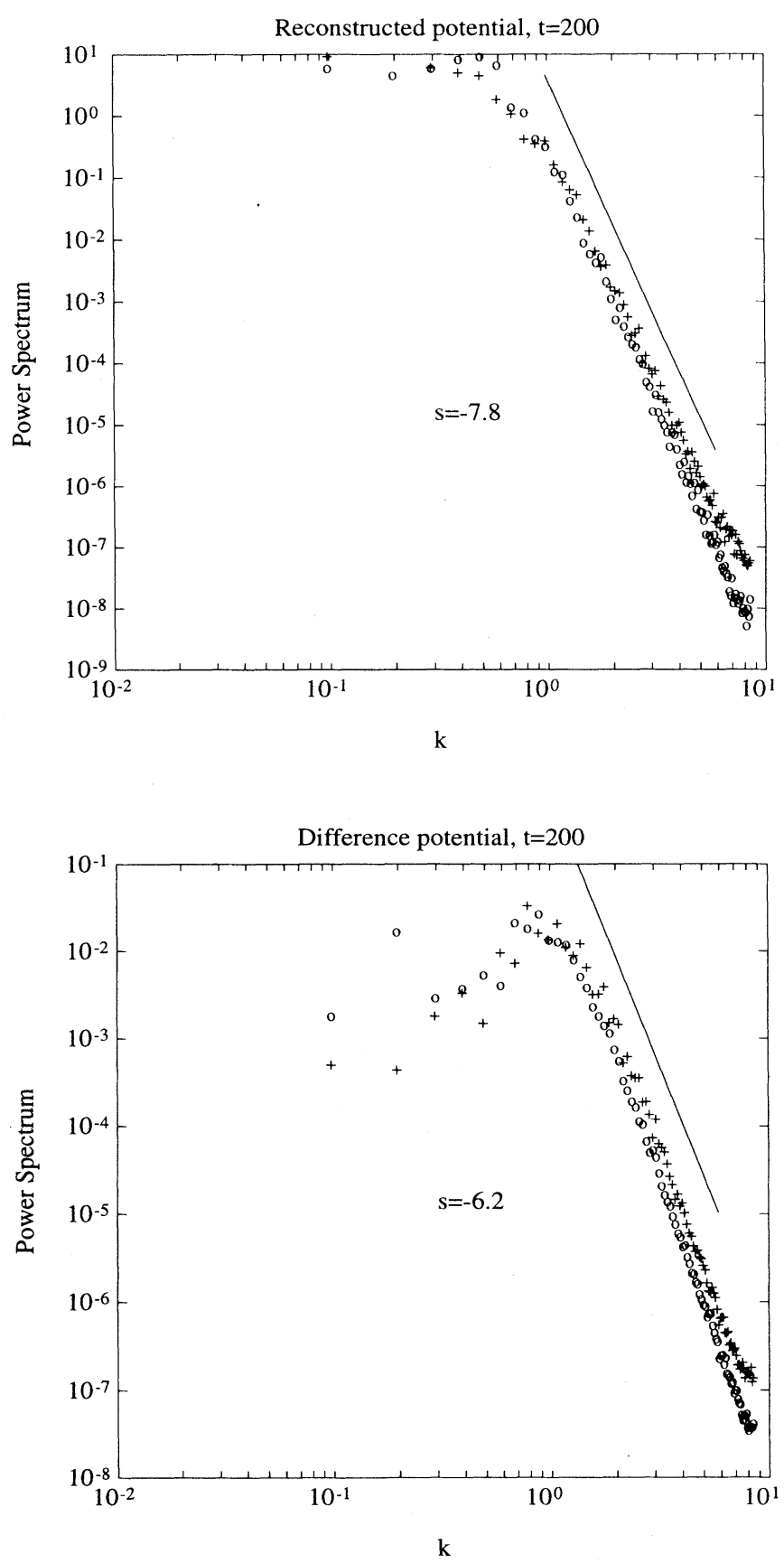

FIG. 22. Power spectrum of the reconstructed (top) and of the difference (bottom) potential of Fig. 21. The reconstructed field shows a slope slightly larger than the intermediate $k$ slope of the original signal. The fluctuating (incoherent) part of the signal has at large $k$, a slope which is closer to the cascade spectrum slope. the sense of the departure from a Gaussian distribution of the vorticity. Obviously, the generalized vorticity of the Hasegawa-Mima equation is no longer conserved in this driven-dissipative case, therefore, as in case (ii) of decaying turbulence, evolution towards non-Gaussian dis-
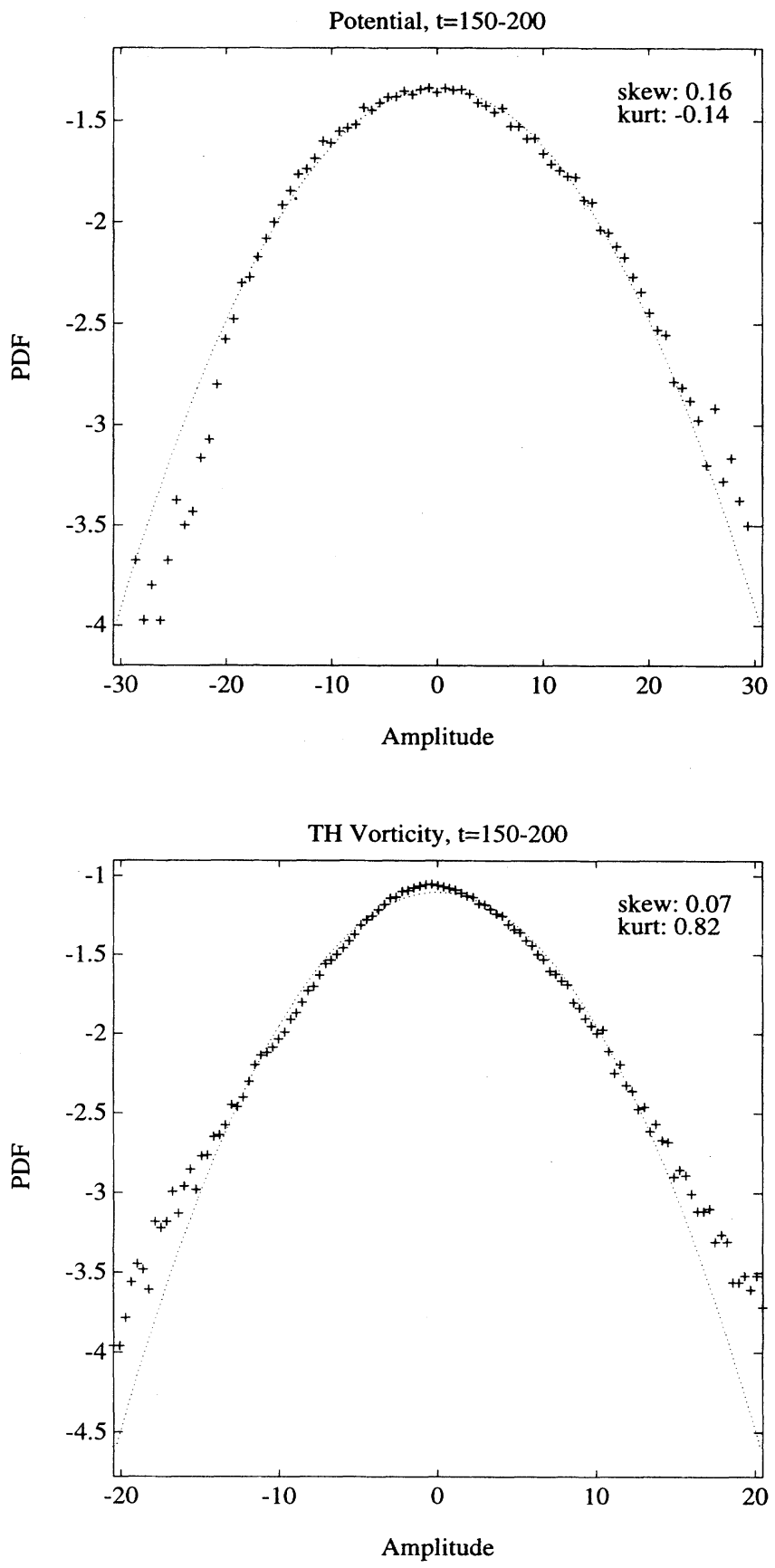

FIG. 23. Histograms of the potential and (generalized) vorticity in the stationary state of driven turbulence. Log-log plot, the reference Gaussian appears as an inverted parabola. The values of the skewness and kurtosis are small, but the $\chi^{2}$ test shows the non-Gaussian character of the distribution of the vorticity. Potential fluctuations are close to Gaussian. The histogram is obtained from the signal in the period between $t=150$ and $t=200$. 
tribution of the vorticity (and its moments) is possible. We show in Fig. 23 the histograms of the potential and the vorticity $L \phi$. The potential fluctuations (time average) are almost Gaussian. However, the vorticity shows a significant departure from Gaussian statistics. The intermittency is related to the coherence introduced by the large vortices, which contain, as found in the POD analysis, a large Fourier spectrum. Moreover, the histogram of the reconstructed potential and vorticity fields (using the first modes of the POD) are almost identical to the full fields. This means that coherent structures are relevant in determining the statistical properties of the system. However, the relation between intermittency and coherent structures is somewhat subtle. Intermittency is effectively related to the presence of such structures, but the mean contribution to non-Gaussian statistics comes from the small-scale fluctuations. Indeed, the vorticity field consists of isolated vortices (which may be identified from the reconstructed potential field) and small structures concentrated in the large gradients surrounding the vortices. The fact that the POD components have a wide Fourier power spectrum is an indication of their richness in spatial scales; then large scales are correlated to small ones. The small fluctuations are then correlated to the coherent structures in space and in time (they are advected by the large vortices). Even if the phases of the small structures are random, their spatial distribution is highly nonuniform, consisting essentially in small filaments of vorticity gradients. We remark that nonGaussian distribution was found in the experiments of the Aditya tokamak [33], in the edge region where dissipative drift turbulence is important.

\section{DISCUSSION AND CONCLUSIONS}

In the first part of this paper we studied the collision of dipoles, as a step in our understanding of the behavior of coherent structures in $2 \mathrm{D}$ turbulent systems. Indeed, dipoles, which are a full localized solution of the Hasegawa-Mima equation, are a suitable model for the investigation of the nonlinear mechanisms of the system.

We observed in the numerical simulations that the dipole interactions show a rich variety of processes ranging from elastic collisions, similar to solitons in one dimension, to very inelastic ones, with merging and splitting of the dipoles, and monopole formation. We introduced a parameter measuring the role of nonlinearity during collision, and allowing us to classify the interactions. In particular, almost elastic collision is obtained when the turnover time of vortices is larger than the collision time: large amplitude and fast structures are very robust and survive to strong perturbations.

The generation and stability of these vortices also depend on their interaction with waves. If the coupling of the vortices and waves is weak, they are relatively stable and can be present for many turnover times. The reason for this stability is that coherent structures are close to a stationary solution of the dynamical equation; there is a strong correlation between potential and gen- eralized vorticity, assuring that nonlinearity is small. On the other hand, they have translation velocities larger than the phase velocity of waves, and thus their coupling is also small.

This picture is confirmed by the study of dipoles when (linear) unstable modes and dissipation are added to the system. If the perturbation is strong enough to stop the vortex, it is destroyed by emission of waves. But, depending on the internal parameters of the dipole, it can survive, being slowly damped or separated into monopoles after collision with other dipoles.

Moreover, these numerical simulations of vortex collisions show that the role of radiation is fundamental. The formation of monopoles is accompanied by the emission of drift waves; even if the monopole structure is close to a stationary solution, while it moves it leaves a wake of drift waves. A theory of vortex interaction should take into account these radiative processes. This is perhaps the reason why a consistent point vortex model of the Hasegawa-Mima equation is not possible, in opposition to the case of Euler equation, where linear waves are missing $[41,42,44]$.

The decaying turbulence in the frame of the HasegawaMima model appears to be essentially different from the 2D hydrodynamic case. An initial Gaussian field evolves to a quasistationary state after a transitory, where, in accordance to the inverse cascade theory, several generations of vortex merging are produced. The quasistationary state, in the sense that spatial and statistical properties change slowly (with respect to linear and turnover times), is reached when the size of vortices stabilizes through breaking of large structures. Therefore, in contrast to the Euler system, we do not observe an approach to an equilibrium state, where the system consists in a pair of vortices occupying the box size. The complexity of the field at long times is related to the appearance of a nonlinear scale, probably related to the values of the (approximate) integrals of motion and to the "extra" degrees of freedom represented by the waves. Indeed, the nonlinear scale (independent to the box size) allows the existence of localized structures (monopoles or dipoles) interacting weakly, and then, these structures can be superposed at random to obtain a complex spatial distribution. On the other hand, although weak, their coupling with waves impedes the condensation to the largest available length scale.

In the case of a very small dissipation term (in our simulations we do not use hyperviscosity), we note the increase of the fluctuation level, but without destroying the large-scale self-organization. The coherent structures, which dominate, from a dynamical point of view, this self-organized state, can be described by a few degrees of freedom, and on the other hand, they contribute to a rather steep slope to the power spectrum. We showed, using the proper orthogonal decomposition, that only four eigenfunctions were sufficient to reconstruct the large scales of the potential field.

Some general features of the decaying turbulence are also present in the case of driven (with instability and dissipation) turbulence: an initial transitory passing through the inverse cascade steps (towards a condensa- 
tion to large scales), but now characterized by the dominance of the unstable modes, and in particular their anisotropy; the transitory is followed by the formation of large vortices, associated with a highly complex distribution of the (generalized) vorticity; finally a stationary state is set up, where vorticity and potential are well correlated. In contrast to the pure decay case, the coherent structures are strongly perturbed and their lifetime is only a few turnover times. However, the basic mechanisms, like merging of equal sign monopoles or breaking of the largest ones, are similar in both cases.

An interesting problem in driven turbulence is the mechanism of saturation of the instability, and the stopping of the inverse cascade of energy, which normally would drive the system to an accumulation of the energy at large scales. We first note that in fact the mere formation of coherent structures invalidates the pure cascade description of turbulence. Indeed, in terms of Fourier modes, correlation of phases and even nonlocal interactions (in Fourier space) are indispensable to vortex emergence. The precise mechanism of saturation of turbulence is not known, but simulations show that one important process is the breaking of large monopoles: this is a direct "cascade" in Fourier space (not necessarily local), opposite to the normal cascade of energy towards large scales. In addition to the breaking of vortices, what is essential in the setup of the stationary state (dissipation is absent at large scales) is the mechanism of nonlinearity depletion, related to the coherent structures, because they establish a relation between potential and vorticity, diminishing by the way the nonlinear part of the equations and then the source of the inverse cascade.

We demonstrate the existence of this correlation between potential and vorticity in the turbulent state, using the technique of conditional averaging. We found that there is no systematic shift between maxima of potential and vorticity, and we obtained, by averaging in space and time, the fields satisfying that their amplitude is larger than a threshold, a strong functional relation, although nonlinear as in the case of the monopoles formed after dipole collision.

One of our most interesting results is the application of the POD to separate the contribution to the Fourier spectrum of (spatial) large scales, the coherent structures, and the random fluctuations. We showed that coherent structures contribute to a power spectrum decaying algebraically with a steep slope, and that of waves following the inverse cascade spectrum. This diagnostic also confirms the view that the self-organized state of plasma drift turbulence consists essentially in a coherent part, which is in some sense of low (dynamical) dimension, and a field of random waves.

Finally we found that the probability distribution of the potential amplitudes follows approximately a Gaussian statistics, while the vorticity is non-Gaussian, which can be interpreted as a manifestation of intermittency. The departure from Gaussian statistics is then not directly related to the presence of coherent structures, but apparently we must take into account the contribution of small scales. Indeed, the potential is much more smooth than the vorticity field to small scales, in particular in the vorticity field there are steep gradients and regions of rather flat profile, which are the main source of intermittency. It would be interesting to establish in a more rigorous way the relation between intermittency and coherent structures, in particular using a spatiotemporal analysis. In fact, an understanding of the mechanism of selforganization of the vorticity field in long-lived and correlated localized structures, superposed to a background field of fluctuations, is essential to a dynamical theory of intermittency.

\section{ACKNOWLEDGMENTS}

The authors gratefully acknowledge helpful discussions with S. Benkadda, T. Dudok de Witt, X. Garbet, J.F. Luciani, and D. Pesme. C.F.F. wishes to thank Université de Provence for financial support and Equipe Turbulence Plasma for kind hospitality. Numerical calculations were partially supported by the Institut Méditerranéen de Technologie and Conseil Régional de Provence.
[1] A. Hasegawa, Adv. Phys. 34, 1 (1985).

[2] M. Brachet, M. Meneguzzi, H. Politano, and P. Sulem, J. Fluid Mech. 194, 333 (1988).

[3] S. Gama, U. Frish, and H. Scholl, J. Sci. Comput. 6, 425 (1991).

[4] J. C. McWilliams, J. Fluid Mech. 219, 361 (1990).

[5] J. C. McWilliams, J. Fluid Mech. 219, 387 (1990).

[6] A. Vincent and M. Meneguzzi, J. Fluid Mech. 255, 1 (1991).

[7] D. Bonn, Y. Couder, P. H. J. van Dam, and S. Douady, Phys. Rev. E 47, R28 (1993).

[8] A. Pouquet, in Spatio-temporal Analysis for Resolving Plasma Turbulence, ESA WPP-047 Proceedings, Aussois 1993, edited by A. Roux, F. Lefeuvre, and D. LeQueau
(European Space Agency, Paris, 1993).

[9] M. B. Isichenko and A. V. Gruzinov, Phys. Plasmas 1, 1802 (1994).

[10] A. K. M. F. Hussain, J. Fluid Mech. 173, 303 (1986).

[11] R. H. Kraichnan and D. Montgomery, Rep. Prog. Phys. 43, 547 (1980).

[12] M. Lesieur, Turbulence in Fluids (Kluwer Academic, Dordrecht, 1990).

[13] W. Horton, Phys. Rep. 192, 1 (1990).

[14] A. Hasegawa and K. Mima, Phys. Rev. Lett. 39, 205 (1977).

[15] A. B. Mikhailovsky, in Nonlinear Phenomena in Plasma Physics and Hydrodynamics, edited by R. Z. Sagdeev (Mir Publishers, Moscow, 1986). 
[16] P. Terry and W. Horton, Phys. Fluids 25, 491 (1982).

[17] P. Terry and W. Horton, Phys. Fluids 26, 106 (1983).

[18] J. A. Crotinger and T. H. Dupree, Phys. Fluids B 4, 2854 (1992).

[19] A. Hasegawa and M. W. Wakatani, Phys. Rev. Lett. 59, 1581 (1987).

[20] W. Horton, Phys. Fluids 29, 1491 (1986).

[21] W. Horton, Phys. Fluids B 1, 524 (1989).

[22] M. Makino, T. Kamimura, and T. Taniuti, J. Phys. Soc. Jpn. 50, 980 (1981).

[23] J. C. McWilliams and N. J. Zabusky, Geophys. Astrophys. Fluid Dynamics 19, 207 (1982).

[24] J. Nycander, J. Plasma Phys. 39, 413 (1988).

[25] S. V. Musylev and G. M. Reznik, Phys. Fluids B 4, 2841 (1992).

[26] R. Robert and J. Somméria, J. Fluid. Mech. 229, 291 (1991).

[27] W. H. Matthaeus, W. T. Stribling, D. Martinez, S. Oughton, and D. Montgomery, Physica (Amsterdam) 51D, 531 (1991).

[28] J. M. Albert, P. L. Similon, and R. N. Sudan, Phys. Fluids B 2, 3032 (1990).

[29] J. L. Lumley, Stochastic Tools in Turbulence (Academic, New York, 1970).

[30] N. Aubry, R. Guyonnet, and R. Lima, J. Stat. Phys. 64, 683 (1991).
[31] S. Benkadda, T. Dudok de Witt, A. D. Verga, A. Sen, ASDEX team, and X. Garbet, Phys. Rev. Lett. 73, 3403 (1994).

[32] T. Huld, A. H. Nielsen, H. L. Pécseli, and J. J. Rasmunssen, Phys. Fluids B 3, 1609 (1991).

[33] R. Jha, P. K. Kaw, S. K. Mattoo, C. V. S. Rao, Y. C. Saxena, and ADITYA Team, Phys. Rev. Lett. 69, 1375 (1992).

[34] G. S. Patterson and S. A. Orszag, Phys. Fluids 14, 2538 (1971).

[35] J. Gazdag, J. Comput. Phys. 20, 196 (1976).

[36] A. B. Mikhailovsky, V. P. Lakhin, L. A. Mikhailovskaya, and O. G. Onishchenko, Zh. Eksp. Teor. Fiz. 86, 2061 (1984) [Sov. Phys. JETP 59, 1198 (1984)].

[37] J. Nycander, Phys. Fluids A 4, 467 (1992).

[38] L. Onsager, Suppl. Nuovo Cimento 6, 279 (1949).

[39] J. Miller, P. B. Weichman, and M. C. Cross, Phys. Rev. A 45, 2328 (1992).

[40] L. J. Campbell and K. O'Neil, J. Stat. Phys. 65, 495 (1991).

[41] N. J. Zabusky and J. C. McWilliams, Phys. Fluids 25, 2175 (1982).

[42] M. Kono and W. Horton, Phys. Fluids B 3, 3255 (1991).

[43] M. Kono and E. Miyashita, Phys. Fluids 31, 326 (1988).

[44] C. Matsuoka and K. Nozaki, Phys. Fluids A 4, 551 (1992). 